\title{
Origin of wide-band IP type II bursts ${ }^{\star}$
}

\author{
S. Pohjolainen ${ }^{1}$, H. Allawi ${ }^{1,2}$, and E. Valtonen ${ }^{3}$ \\ 1 Tuorla Observatory, Department of Physics and Astronomy, University of Turku, Piikkiö, Finland \\ e-mail: silpoh@utu.fi \\ 2 Department of Physics, Thi Qar University, Nasryia, Iraq \\ e-mail: habeeb.allawi@utu.fi \\ 3 Space Research Laboratory, Department of Physics and Astronomy, University of Turku, Turku, Finland \\ e-mail: eino.valtonen@utu.fi
}

Received 2 November 2012 / Accepted 6 July 2013

\begin{abstract}
Context. Different types of interplanetary (IP) type II bursts have been observed, where the more usual ones show narrow-band and patchy emissions, sometimes with harmonics, and which at intervals may disappear completely from the dynamic spectrum. The more unusual bursts are wide-band and diffuse, show no patches or breaks or harmonic emission, and often have long durations. Type II bursts are thought to be plasma emission, caused by propagating shock waves, but a synchrotron-emitting source has also been proposed as the origin for the wide-band type IIs.

Aims. Our aim is to find out where the wide-band IP type II bursts originate and what is their connection to particle acceleration. Methods. We analyzed in detail 25 solar events that produced well-separated, wide-band IP type II bursts in 2001-2011. Their associations to flares, coronal mass ejections (CMEs), and solar energetic particle events (SEPs) were investigated.

Results. Of the 25 bursts, 18 were estimated to have heights corresponding to the CME leading fronts, suggesting that they were created by bow shocks ahead of the CMEs. However, seven events were found in which the burst heights were significantly lower and which showed a different type of height-time evolution. Almost all the analyzed wide-band type II bursts were associated with very high-speed CMEs, originating from different parts of the solar hemisphere. In terms of SEP associations, many of the SEP events were weak, had poor connectivity due to the eastern limb source location, or were masked by previous events. Some of the events had precursors in specific energy ranges. These properties and conditions affected the intensity-time profiles and made the injection-timebased associations with the type II bursts difficult to interpret. In several cases where the SEP injection times could be determined, the radio dynamic spectra showed other features (in addition to the wide-band type II bursts) that could be signatures of shock fronts. Conclusions. We conclude that in most cases (in 18 out of 25 events) the wide-band IP type II bursts can be plasma emission, formed at or just above the CME leading edge. The results for the remaining seven events might suggest the possibility of a synchrotron source. These events, however, occurred during periods of high solar activity, and coronal conditions affecting the results of the burst height calculations cannot be ruled out. The observed wide and diffuse emission bands may also indicate specific CME leading edge structures and special shock conditions.
\end{abstract}

Key words. Sun: radio radiation - Sun: coronal mass ejections (CMEs) - shock waves - plasmas

\section{Introduction}

Shock waves can be observed in the solar corona and interplanetary (IP) space by using radio observations of type II bursts. Electrons accelerated by the shock produce Langmuir waves in the surrounding plasma, and these eventually turn into radio waves (e.g., Melrose 1980). Type II bursts are frequencydrifting emissions, with typical drift rates of $1.0-0.1 \mathrm{MHz} \mathrm{s}^{-1}$ in the corona (metric emission) and much less in the IP space. The drift rates reflect the decreasing density in the solar atmosphere, as the plasma frequency changes as $f_{\mathrm{p}}=9000 \sqrt{n_{\mathrm{e}}}(f$ in $\mathrm{Hz}$ and electron density $n_{\mathrm{e}}$ in $\mathrm{cm}^{-3}$ ). Type II bursts are often identified with fundamental and harmonic plasma emission bands, although at decimeter-meter waves the fundamental band and at decameter-hectometer (DH) waves the harmonic band can be undetectable because of plasma processes. Sometimes the emission is also band-split (Vrsnak et al. 2001).

^ Figures 2-26 and Table 4 are available in electronic form at http://www . aanda.org
There is a long-standing debate over the drivers or launchers of the shock waves that produce type II bursts. Generally, it is agreed that fast-propagating coronal mass ejections (CMEs) can create propagating shock waves, either as bow shocks at the nose of the CME or as driven waves at the CME flanks. In the low corona there is also the possibility that flare-ignited blast waves propagate outward and produce type II bursts (see review on coronal shocks in, e.g., Warmuth 2007).

Type II bursts at DH wavelengths are usually attributed to bow shocks ahead of CMEs, but in many well-analyzed cases of type II-CME events the calculated radio burst speeds and burst heights do not correspond to the CME speeds and the CME front heights (e.g., Cho et al. 2007; Vrsnak et al. 2006; Kim et al. 2009). This has been explained by projection effects of the white-light CME structures and/or unreliable density estimates for the type II burst emissions, for example. Propagating shocks at the flanks of a CME would also show speeds and heights different from those of a CME bow shock. In the absence of radio imaging in $\mathrm{DH}$ waves and because of the rare imaging facilities in metric waves, the true type II burst locations often remain unknown. 

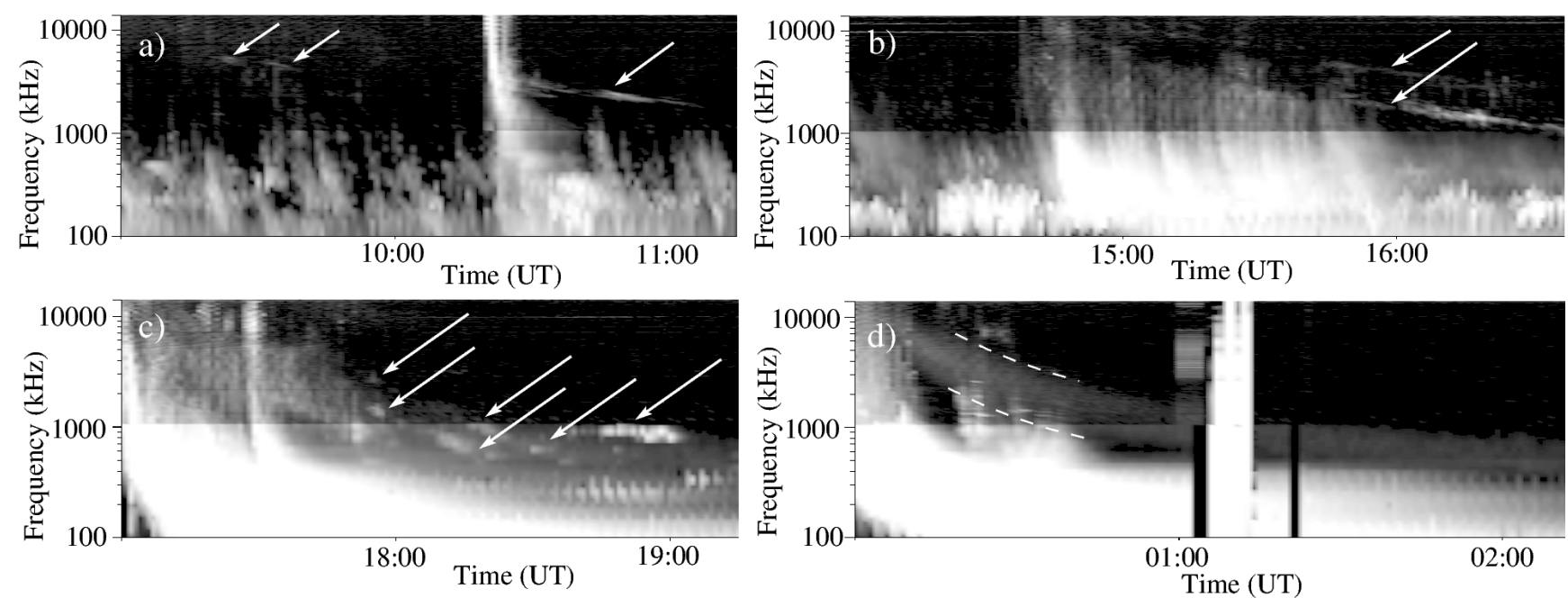

Fig. 1. Type II bursts with different characteristics, observed by the Wind WAVES instruments and plotted here in the $14 \mathrm{MHz}-100 \mathrm{kHz}$ frequency range. a) A complex type II burst on 19 January 2005 with intermittent emission. b) A type II burst on 23 August 2005 that shows narrow-band emission at fundamental and harmonic plasma frequencies. c) Chaotic, intermittent, and patchy type II burst emission on 22 August 2005 . d) A wide-band type II burst on 3 December 2004 (our event No. 16); the width of the emission band is indicated with dashed lines (the frequency of the band center was used for the height calculations, see text for details). All four type II bursts were associated with high-speed halo-type CMEs.

Type II bursts are often observed in the IP space as narrowband and patchy emissions, which at intervals can disappear completely. Some examples are shown in Figs. 1a-c. Another type of IP type II burst has also been reported, characterized by smooth and diffuse wide-band emission lanes that show no patches or breaks in their emission (an example is shown in Fig. 1d), and which usually start below $\sim 4 \mathrm{MHz}$ and can last for hours (Cairns et al. 2000; Cane \& Erickson 2005, and references therein). According to Mann et al. (1995) the instantaneous bandwidth of a type II burst reflects the density jump across the associated shock wave. It has also been suggested that the fundamental plasma emission is generated in the downstream region of the shock, and its harmonic in the upstream region (Hoang et al. 1992). Since the wide-band IP type II bursts show only one diffuse and broad emission band with no harmonic emission, the density structure within the shocked region could be reflected in the emission characteristics.

Wide-band type II bursts have been associated mainly with fast halo-type CMEs, which suggests that the propagating shocks are bow shocks ahead of the CMEs. However, the burst on 17-18 June 2003 that was analyzed in detail by Bastian (2007) was suggested to be not due to plasma radiation at all, but to synchrotron emission from near-relativistic particles trapped in the CME magnetic field (trapping location not specified). The main reason for this suggestion was that the type II burst frequency drift was inconsistent with that expected from plasma radiation associated with a CME-driven shock. Both the Bastian (2007) event and the wide-band event on 24 September 2001, analyzed by Vourlidas et al. (2007a,b), were also reported as being associated with solar energetic particle (SEP) events.

Since shock waves are considered to be the main SEP accelerators, the origin of the shock, the efficiency of particle acceleration, and the time of first injection (i.e., first acceleration) are of interest. Especially large particle events and low-frequency type II emissions are known to be associated with fast and wide CMEs (Cane \& Stone 1982; Cane et al. 1987; Gopalswamy et al. 2008a), although not all fast and wide CMEs produce DH type II bursts or SEPs (Gopalswamy et al. 2001, 2008b). As wide-band type II bursts have been reported as having an association with fast-propagating halo-type CMEs, which most likely create bow shocks at the leading fronts and accelerate SEPs, they should also have an association with SEP events. If no SEPs are observed and the wide-band type II burst heights do not match the CME bow shock heights, other scenarios for the type II emission should also be considered.

The motivation of our study is to find out where the diffuse wide-band type II bursts originate and how they are related to flares, CMEs, and SEPs. Section 2 describes the selection criteria for our data set and how the measurements and estimates were done. Section 3 gives the main results from the analysis, and in Sect. 4 we discuss these results and present the conclusions.

\section{Data analysis}

We started the analysis by going through the listed IP type II events from 1996-2011, observed by the Radio and Plasma Wave Investigation (WAVES) instruments (Bougeret et al. 1995) onboard the Wind satellite. The burst lists and spectral plots, prepared by Michael L. Kaiser, are available at http://lep694. gsfc.nasa.gov/waves/data_products.html. The lists for 1996-2011 consist of a total of 565 entries, although some intermittent bursts may have been listed as separate bursts and some type IV bursts may have been mixed with type II bursts. Wideband type II bursts were first identified visually from the spectral plots, after which the IDL save files for each day were inspected more carefully.

Wide-band type II emission is usually fainter in intensity than the more common narrow-band and patchy type II emission and, therefore, more difficult to detect. Very often intense type III burst emission (fast particle beams created in the eruption phase) block out the start of the type II burst emission, as in the event shown in Fig. 1d, making it impossible to define the true start time or frequency. Some type II events were dismissed from our sample because of their inadequate time interval, as comparison to CME heights and speeds was not possible.

Events where multiple CMEs were observed to propagate simultaneously in the same or different directions were also omitted from the data set, bacause of the uncertainties in the association of type II burst heights (i.e., when the radio burst could be associated to more than one CME front or to the flanks of 
Table 1. List of analyzed diffuse wide-band type II bursts found in the 1996-2011 radio spectral data.

\begin{tabular}{|c|c|c|c|c|}
\hline No. & $\begin{array}{c}\text { Date } \\
\text { (yyyy-mm-dd) }\end{array}$ & $\begin{array}{l}\text { Visible first at } \\
\text { frequency band }\end{array}$ & $\begin{array}{c}\text { Identified } \\
\text { until }\end{array}$ & Comments \\
\hline 1 & 2001-09-24 & $3 \mathrm{MHz}-500 \mathrm{kHz}$ & $80 \mathrm{kHz}$ & Complex start, very wide band, Vourlidas et al. event \\
\hline 2 & 2002-01-14 & $1 \mathrm{MHz}-500 \mathrm{kHz}$ & $180 \mathrm{kHz}$ & Unclear start near $1 \mathrm{MHz}$ \\
\hline 3 & 2002-08-16 & $8-4 \mathrm{MHz}$ & $\sim 1 \mathrm{MHz}$ & Other shock structures appear $\sim 1 \mathrm{MHz}$, could be two separate lanes \\
\hline 4 & 2002-11-09 & $14-6 \mathrm{MHz}$ & $\sim 1 \mathrm{MHz}$ & Blends with other emission below $1 \mathrm{MHz}$ \\
\hline 5 & 2002-11-10 & $4-1.8 \mathrm{MHz}$ & $400 \mathrm{kHz}$ & Blends with other emission below $400 \mathrm{kHz}$ \\
\hline 6 & 2003-05-28 & $1 \mathrm{MHz}-500 \mathrm{kHz}$ & $150 \mathrm{kHz}$ & Complex start, blends with other emission below $150 \mathrm{kHz}$, another lane at higher freq. \\
\hline 7 & 2003-05-29 & $8-3.5 \mathrm{MHz}$ & $700 \mathrm{kHz}$ & Blends with other emission below $700 \mathrm{kHz}$ \\
\hline 8 & 2003-05-31 & $1.2 \mathrm{MHz}-600 \mathrm{kHz}$ & $180 \mathrm{kHz}$ & Unclear start near $1 \mathrm{MHz}$ \\
\hline 9 & $2003-06-17 / 18$ & $8-4 \mathrm{MHz}$ & $200 \mathrm{kHz}$ & Strong band, suggested synchrotron source, Bastian event \\
\hline 10 & 2004-04-06 & 7.5-4 MHz & $250 \mathrm{kHz}$ & Very faint below $1 \mathrm{MHz}$ \\
\hline 11 & 2004-04-11 & $10-5 \mathrm{MHz}$ & $600 \mathrm{kHz}$ & Blends with other emission below $2 \mathrm{MHz}$ \\
\hline 12 & 2004-07-25 & $1.4 \mathrm{MHz}-600 \mathrm{kHz}$ & $150 \mathrm{kHz}$ & Start near $1 \mathrm{MHz}$, another lane at higher frequencies \\
\hline 13 & 2004-07-29 & $1 \mathrm{MHz}-500 \mathrm{kHz}$ & $200 \mathrm{kHz}$ & Start near $1 \mathrm{MHz}$, blends with other emission (bright patches) below $300 \mathrm{kHz}$ \\
\hline 14 & 2004-07-31 & $900-600 \mathrm{kHz}$ & $200 \mathrm{kHz}$ & Narrower band, blends with other emission below $200 \mathrm{kHz}$ \\
\hline 15 & 2004-09-12 & $2-1 \mathrm{MHz}$ & $300 \mathrm{kHz}$ & Band edge brightens below $1 \mathrm{MHz}$, another narrow lane at higher frequencies \\
\hline 16 & $2004-12-02 / 03$ & $10-5 \mathrm{MHz}$ & $300 \mathrm{kHz}$ & Faint below $300 \mathrm{kHz}$, blends with other emission \\
\hline 17 & 2005-01-15 & $1.2 \mathrm{MHz}-600 \mathrm{kHz}$ & $200 \mathrm{kHz}$ & Blends with other emission below $400 \mathrm{kHz}$ \\
\hline 18 & $2005-01-15 / 16$ & $700-300 \mathrm{kHz}$ & $180 \mathrm{kHz}$ & Complex start, another lane at higher frequencies \\
\hline 19 & 2005-06-03 & $1 \mathrm{MHz}-600 \mathrm{kHz}$ & $200 \mathrm{kHz}$ & Blends with other emission below $200 \mathrm{kHz}$, narrow lane with patches \\
\hline 20 & 2005-07-27 & $1.6 \mathrm{MHz}-800 \mathrm{kHz}$ & $300 \mathrm{kHz}$ & Blends with other emission below $400 \mathrm{kHz}$, gets narrower \\
\hline 21 & 2005-07-30 & $4-1.5 \mathrm{MHz}$ & $500 \mathrm{kHz}$ & Blends with other emission below $500 \mathrm{kHz}$, could be two separate lanes \\
\hline 22 & 2005-09-09 & $5-2 \mathrm{MHz}$ & $200 \mathrm{kHz}$ & Bright patches below $1 \mathrm{MHz}$, other structures at higher frequencies \\
\hline 23 & 2005-09-10 & $14+\mathrm{MHz}$ & $200 \mathrm{kHz}$ & Bright patches below $1.5 \mathrm{MHz}$ \\
\hline 24 & 2011-03-07 & $1 \mathrm{MHz}-500 \mathrm{kHz}$ & $200 \mathrm{kHz}$ & Mixed with bursty emission \\
\hline 25 & 2011-09-22 & $1.1 \mathrm{MHz}-400 \mathrm{kHz}$ & $200 \mathrm{kHz}$ & Mixed with bursty emission \\
\hline
\end{tabular}

more than one CME). Furthermore, in multiple CME events the burst height calculations can be affected by the disturbed solar atmospheric densities if shocks are propagating in the wake of earlier CMEs. However, events with well-separated characteristics in speed and direction were still accepted. To test if a preceding (non-simultaneous) CME with a large angular width $\left(>100^{\circ}\right)$ could be a precondition for the formation of a wideband type II burst, we also calculated the time difference between the launch of a preceding CME and our type II-associated CME (see Sect. 2.3).

From the 1996-2000 event listings, only one wide-band IP type II burst was found. Unfortunately, this event occurred during the Solar and Heliospheric Observatory (SOHO) mission break and there was no Large Angle and Spectrometric Coronagraph (LASCO) data to compare with. In addition the 2006-2010 period of low solar activity produced no wide-band type II bursts that could be analyzed. In total, 25 wide-band type II bursts that fulfilled the selection criteria were found from the years 2001-2005 and 2011, and were then analyzed. The two previously mentioned events from Bastian (2007) and Vourlidas et al. (2007a) were also found in the data set. The 25 events are listed in Table 1, giving the frequency band where the type II burst was first identified, and the lowest frequency where the band could still be identified before it vanished or got mixed with other emissions.

The identified bursts were then compared with the entries in the SOHO LASCO CME Catalog ${ }^{1}$. For the CME heights we used the CME leading edge heights determined from the

\footnotetext{
1 http://cdaw.gsfc.nasa.gov/
}

LASCO C2 and C3 coronagraph (Brueckner et al. 1995) images and published in the LASCO CME Catalog. The LASCO C3 field of view extends to $32 R_{\odot}$, which corresponds to a plasma frequency of about $200 \mathrm{kHz}$. For many fast CMEs the last observation point was much less than $32 R_{\odot}$, which in some cases complicated the comparison to the calculated type II burst heights.

The type II burst heights were calculated at the times of the CME images, when possible. The method of determining type II burst heights using atmospheric models is described in Pohjolainen et al. (2007), for example. Their Table 1 also compares the heights calculated using different atmospheric models. The hybrid density model of Vrsnak et al. (2004) was selected for our analysis because it was developed to merge the highdensity low-corona models to the low-density IP models without breaks or discrepancies. It is basically a five-times Saito model (see the basic one-fold model in Saito 1970) combined to the Leblanc et al. (1998) model with small modifications. The model gives an electron density of $7 \mathrm{~cm}^{-3}$ near $1 \mathrm{AU}$. This is the density value observed during solar minimum (during activity maximum the value can be ten times this high) and so these model densities should work well with events observed during solar activity minimum.

Because the analyzed type II burst emission lanes had a wide bandwidth, the calculations were done using the emission lane center-frequency, i.e., if emission was observed at $2-4 \mathrm{MHz}$ the calculation frequency was $3 \mathrm{MHz}$. Because the observed radio emission can be assumed to be slightly above the electron plasma frequency (e.g., Mann et al. 1995), using the lane center as $f_{\mathrm{p}}$ should give reasonable results. 
Table 2. Diffuse wide-band type II bursts with heights in agreement with CME leading front heights.

\begin{tabular}{|c|c|c|c|c|c|c|c|c|c|c|c|}
\hline No. & $\begin{array}{c}\text { Date } \\
\text { (yyyy-mm-dd) }\end{array}$ & $\begin{array}{c}\text { Flare } \\
\text { start } \\
\text { (UT) }\end{array}$ & $\begin{array}{l}\text { Flare } \\
\text { GOES } \\
\text { class }\end{array}$ & $\begin{array}{l}\text { Flare/ } \\
\text { CME } \\
\text { source } \\
\text { region }\end{array}$ & $\begin{array}{l}\text { CME } \\
\text { obs. } \\
\text { (UT) }\end{array}$ & $\begin{array}{c}\text { CME } \\
\text { height }^{a} \\
\left(R_{\odot}\right)\end{array}$ & $\begin{array}{l}\text { Type II } \\
\text { height }^{a} \\
\left(R_{\odot}\right)\end{array}$ & $\begin{array}{c}\text { CME } \\
\text { speed }^{b} \\
\left(\mathrm{~km} \mathrm{~s}^{-1}\right)\end{array}$ & $\begin{array}{l}\text { Type II } \\
\text { speed }^{b} \\
\left(\mathrm{~km} \mathrm{~s}^{-1}\right)\end{array}$ & $\begin{array}{c}\text { SEP } \\
\text { injection }^{c} \\
\text { time } \\
\text { (UT) }\end{array}$ & $\begin{array}{c}\text { CME } \\
\text { height } \\
\text { at first } \\
\text { injection }^{d} \\
\left(R_{\odot}\right)\end{array}$ \\
\hline 4 & $2002-11-09$ & 13:08 & M4.6 & S12W29 & $13: 31$ & 4.2 & 4.4 & $1790(\mathrm{~A})$ & 2300 (D) & $13: 05 \pm 9$ & $\sim 0$ \\
\hline 5 & $2002-11-10$ & 03:04 & M2.4 & S12W37 & 03:42 & 6.9 & 7.1 & 1675 (D) & 1490 (D) & NP & \\
\hline 6 & $2003-05-28$ & $00: 17$ & X3.6 & S08W22 & $01: 42$ & 9.9 & 14.0 & $1450(\mathrm{~A})$ & $1290(\mathrm{~A})$ & $\mathrm{U} 00: 15 \pm 11$ & $\sim 0$ \\
\hline 7 & 2003-05-29 & $00: 51$ & $\mathrm{X} 1.2$ & S06W37 & $01: 27$ & 5.0 & 6.6 & 1470 (D) & 850 (A) & high bg & \\
\hline 10 & 2004-04-06 & $12: 30 / 13: 15$ & M2.4 & S18E15 & $13: 31$ & 3.9 & 4.5 & $655(\mathrm{~A})$ & $960(\mathrm{~A})$ & - & \\
\hline 11 & 2004-04-11 & $03: 54$ & C9.6 & S14W47 & $04: 30$ & 4.8 & 5.0 & 1870 (D) & $1840(-)$ & $04: 06 \pm 6$ & $\sim 0$ \\
\hline 12 & 2004-07-25 & $13: 37 / 14: 19$ & M1.1 & N08W33 & $15: 30^{1}$ & 6.4 & 10.9 & 1550 (D) & $1160(\mathrm{~A})$ & $15: 04 \pm 6$ & $\sim 4$ \\
\hline 13 & 2004-07-29 & $11: 42$ & $\mathrm{C} 2.1$ & W-limb & $14: 18^{2}$ & 14.3 & 16.5 & $1280(\mathrm{~A})$ & 980 (D) & NP & \\
\hline 14 & 2004-07-31 & $05: 16$ & $\mathrm{C} 8.4$ & W-limb & $07: 42$ & 11.5 & 13.6 & $1220(\mathrm{~A})$ & 1040 (D) & high bg & \\
\hline 15 & 2004-09-12 & 00:04 & M4.8 & N04E42 & $01: 24$ & 6.7 & 9.3 & $1370(\mathrm{~A})$ & $1010(\mathrm{~A})$ & high bg & \\
\hline 16 & $2004-12-02 / 03$ & $23: 44$ & M1.5 & N09E03 & $00: 25$ & 4.2 & 6.1 & 1350 (D) & 1550 (D) & precursor $^{3}$ & \\
\hline 17 & $2005-01-15$ & $05: 54$ & M8.6 & N16E04 & $06: 42$ & 7.9 & 11.7 & 2120 (D) & 1800 (D) & $06: 20 \pm 4$ & $\sim 4$ \\
\hline 18 & $2005-01-15 / 16$ & $21: 54 / 22: 25$ & X2.6 & N15W05 & $23: 42$ & 16.5 & 16.5 & 2600 (D) & 2100 (D) & $22: 52 \pm 5$ & $\sim 4$ \\
\hline 19 & 2005-06-03 & $11: 51$ & M1.0 & E-limb & $13: 38$ & 15.3 & 16.5 & 1590 (D) & $1450(-)$ & NP & \\
\hline 20 & 2005-07-27 & $04: 33$ & M3.7 & E-limb & $05: 18$ & 6.7 & 9.3 & 1930 (D) & $970(\mathrm{~A})$ & NP & \\
\hline 21 & 2005-07-30 & 05:03/06:17 & $\mathrm{X} 1.3$ & N12E60 & $06: 50$ & 5.6 & 8.2 & 2020 (D) & 2550 (D) & NP & \\
\hline 22 & 2005-09-09 & $17: 32 / 19: 13$ & X6.2 & S12E67 & 20:00 & 5.6 & 5.9 & 2590 (D) & $1610(\mathrm{~A})$ & high bg & \\
\hline 23 & 2005-09-10 & $19: 10 / 21: 30$ & X2.1 & S13E47 & $21: 52$ & 4.0 & 4.9 & 2030 (D) & 2030 (D) & high bg & \\
\hline
\end{tabular}

Notes. ${ }^{(a)}$ Heliocentric CME (LASCO CME Catalog) and radio type II burst heights are given for a time when both could be observed and measured for the first time (see text for details for the height determination methods). ${ }^{(b)}$ Speeds have been calculated using the heights given in Cols. 7 and 8 and the next observed heights, (A) stands for acceleration and (D) for deceleration. ${ }^{(c)}$ SEP injection times were estimated with the VDA method when possible, $\mathrm{U}$ stands for uncertain, NP for analysis not possible, and high bg for events possibly masked by high background. ${ }^{(d)}$ Heliocentric CME height determined from the data in the LASCO CME Catalog, at the time of SEP injection from VDA analysis. (1) CME accelerates or overtakes earlier event near 15:10 UT, at $5 R_{\odot} \cdot{ }^{(2)}$ CME accelerates during LASCO data gap 12:54-14:18 UT. ${ }^{(3)}$ Low energy precursor, higher energies rise later.

Flare characteristics were obtained from the National Oceanic and Atmospheric Administration (NOAA) National Geophysical Data Center (NGDC) solar data archives. Geostationary Operational Environmental Satellites (GOES) $\mathrm{X}$-ray data were also plotted, to check these listings and to identify multiple overlapping flare events.

The SOHO Energetic and Relativistic Nuclei and Electron (ERNE) instrument (Torsti et al. 1995) proton data were then investigated to find SEP events associated with the wide-band IP type II bursts. In cases where clear SEP associations were found, energetic particle first injection times (i.e., times when energetic particles could have been accelerated close to the Sun and been released into open magnetic field lines connected to the observation site) were calculated using a fixed 1.2 AU path length for the SEPs. When possible, the obtained times were re-checked with the velocity dispersion analysis (VDA) method (see, e.g., Lintunen \& Vainio 2004; Huttunen et al. 2005; Kahler \& Ragot 2006). For VDA, ERNE one-minute average proton intensities in 20 energy channels were used, covering the proton energy range from $1.58 \mathrm{MeV}$ to $131 \mathrm{MeV}$. Bacause of the nature of the SEP events (see descriptions in Sect. 2.2), VDA could not be applied in all events and in some events where VDA was applied it was not possible to determine event onset times in all 20 energy channels.

\subsection{Wide-band IP type II bursts and associated events}

The identification of a diffuse emission lane can sometimes be difficult as other types of radio emission structures can be superposed over it, especially at the beginning of the burst. Eventually at long wavelengths it can also mix with the bursty auroral kilometric radiation (AKR). For many wide-band type II bursts it is impossible to determine a start time as the emission gets mixed with the intense type III burst activity during the flare impulsive phase, and an end time may be equally hard to define if the emission band is only partially visible. The analyzed 25 wide-band type II bursts are shown in Figs. 2-26 (online data only). The radio bursts in the dynamic spectra present variable characteristics like high-frequency or low-frequency starts, short or long durations, superposed bursty emissions and/or very smooth sharpedged bands. In some cases additional separate type II lanes are also present even if we had excluded events with simultaneous multiple CMEs.

From Table 1 it can be observed that some wide-band type II bursts were already visible at the highest Wind WAVES frequencies (see for example event No. 23 in Fig. 24), at about $14 \mathrm{MHz}$, but some did not become identifiable until about $1 \mathrm{MHz}$. In most cases the start was hidden by intense type III burst emission, but it appears that some wide-band type II bursts were really formed near the $1 \mathrm{MHz}$ center frequency (see for example the low-frequency start in Fig. 14, event No. 13). As the WAVES dynamic spectrum consists of observations with two different instruments, RAD1 and RAD2, the change in instrument sensitivity is visible near $1 \mathrm{MHz}$ and can affect the type II identification especially if the bursts are faint. A wide emission band (lane) usually means a bandwidth of about $\Delta f / f \approx 1$, so for example at $f=1 \mathrm{MHz}$ the emission lane should be about $1 \mathrm{MHz}$ wide. This was also verified in our data set.

Tables 2 and 3 present the main characteristics of the analyzed wide-band IP type II bursts and associated events. We list the associated flare start time, flare intensity (GOES class), and source region location. Some events showed ongoing flaring 
Table 3. Diffuse wide-band type II bursts with decreasing heights compared to the CME front heights.

\begin{tabular}{|c|c|c|c|c|c|c|c|c|c|c|c|}
\hline No. & $\begin{array}{c}\text { Date } \\
\text { (yyyy-mm-dd) }\end{array}$ & $\begin{array}{l}\text { Flare } \\
\text { start } \\
\text { (UT) }\end{array}$ & $\begin{array}{l}\text { Flare } \\
\text { GOES } \\
\text { class }\end{array}$ & $\begin{array}{c}\text { Flare/ } \\
\text { CME } \\
\text { source } \\
\text { region }\end{array}$ & $\begin{array}{l}\text { CME } \\
\text { obs. } \\
\text { (UT) }\end{array}$ & $\begin{array}{c}\mathrm{CME} \\
\text { height }^{a} \\
\left(R_{\odot}\right)\end{array}$ & $\begin{array}{l}\text { Type II } \\
\text { height }^{a} \\
\left(R_{\odot}\right)\end{array}$ & $\begin{array}{c}\text { CME } \\
\text { speed }^{b} \\
\left(\mathrm{~km} \mathrm{~s}^{-1}\right)\end{array}$ & $\begin{array}{l}\text { Type II } \\
\text { speed }^{b} \\
\left(\mathrm{~km} \mathrm{~s}^{-1}\right)\end{array}$ & $\begin{array}{c}\text { SEP } \\
\text { injection }^{c} \\
\text { time } \\
\text { (UT) }\end{array}$ & $\begin{array}{c}\text { CME } \\
\text { height } \\
\text { at first } \\
\text { injection }^{d} \\
\left(R_{\odot}\right)\end{array}$ \\
\hline 1 & 2001-09-24 & $9: 32 / 10: 20$ & $\mathrm{X} 2.6$ & S16E23 & $11: 42$ & 17.5 & 15. & 2540 (A) & 770 (D) & $\mathrm{U} 10: 31 \pm 8$ & $\sim 3$ \\
\hline 2 & 2002-01-14 & 04:52/05:29 & M4.4 & W-limb & 07:30 & 16.1 & 13.3 & 1660 (A) & 1620 (D) & $\mathrm{U} 07: 56 \pm 48$ & $\sim 13-26$ \\
\hline 3 & $2002-08-16$ & 11:32/11:58 & M5.2 & S14E20 & $12: 30$ & 3.7 & 4.9 & 1740 (D) & $970(-)$ & $\mathrm{U} 14: 20 \pm 11$ & $\sim 19$ \\
\hline 8 & 2003-05-31 & 02:13 & M9.3 & S07W65 & $04: 18$ & 19.6 & 15.6 & 1820 (D) & 1240 (A) & $\mathrm{U} 02: 30 \pm 4$ & $\sim 3$ \\
\hline 9 & 2003-06-17/18 & $22: 27$ & M6.8 & S07E55 & $23: 18$ & 7.0 & 7.3 & 1930 (D) & 1740 (D) & high bg & \\
\hline 24 & 2011-03-07 & 43 & M3.7 & W-limb & $21: 42$ & 21.3 & 11.1 & 2030 (D) & 970 (D) & $20: 27 \pm 6$ & $\sim 8$ \\
\hline 25 & 2011-09-22 & $10: 29$ & X1.4 & E-limb & $12: 30$ & 20.4 & 12.6 & 1605 (D) & 580 (D) & high bg & \\
\hline
\end{tabular}

Notes. ${ }^{(a)(b)(c)(d)}$ as in Table 2.

activity at the time when the associated flare was listed as starting or the flare was a double-peak flare. These are listed with two flare start times in Tables 2 and 3.

The CME and type II burst heights are listed for the first time when both of them could be observed and measured, and the speeds have been calculated using these and the next observed CME and simultaneous type II heights. However, many of the CMEs could be observed before the wide-band type II lanes were identified, and these CME height-times are listed for each event in Figs. 2-26. With our method we may interpolate between observation points, but we do not extrapolate backwards the CME and type II heights, thus minimizing possible errors due to acceleration/deceleration before both emissions are observed. Acceleration and deceleration during observations are also noted.

In some cases the calculated type II heights were well below the CME front heights and/or the radio bursts also showed considerable deceleration in speed, while the CME front speeds did not decrease. Tables 2 and 3 present the analyzed 25 bursts divided into two separate groups, based on the agreement (18 events) or disagreement ( 7 events) of the time evolution of the type II burst heights with CME leading edge heights.

Coronagraph images from LASCO show that most of the associated CMEs had a symmetrical structure, with a wide and smooth leading front (see Figs. 2-26). Exceptions to this were events No. 6 (Fig. 7), No. 24 (Fig. 25), and No. 25 (Fig. 26), which showed very fragmented white-light structures. In many cases there was also a diffuse bright region ahead of a more pronounced CME leading front (see a good example in Fig. 8, event No. 7). Of the 25 CMEs, 22 were full halos and 3 had a large angular width (>197 degrees).

Figure 27 shows an example of a type II event (10 September 2005, event No. 23, listed in Table 2) where the burst emission lane can be followed through the Wind WAVES dynamic spectrum from $14 \mathrm{MHz}$ to about $200 \mathrm{kHz}$, where it starts to blend with AKR emission. The height comparison for this event shows that the type II burst was slightly ahead of the CME front when first observed, and then slightly behind at larger heights at later times (Fig. 27, bottom panel, type II heights marked with circles and CME heights marked with boxes). Because of the uncertainties in atmospheric density models and the possible errors in determining the frequency of the lane center, especially at low frequencies, we estimate that the apparent small difference in heights is not necessarily real and the type II burst was most likely formed by a bow shock at the CME leading front.

An example of a different type of height evolution is shown in Fig. 28 (24 September 2001, event No. 1, listed in Table 3).
The difference between the CME front height and the type II burst height is increasing as the type II burst is propagating more slowly and decelerating as well.

The data for the other events in Table 3 suggest that at the beginning of the burst (at heights $\leq 10 R_{\odot}$ ) the type II heights may agree with the CME front heights, but at later times the height difference becomes more explicit. The wide-band type II burst shown in Fig. 28 is also an example of a burst where the emission lane is observed only some time after the burst start, as other emission structures mask the band edges and the width of the band cannot be determined at the start, although the diffuse emission is already visible.

\subsection{SEP association for the wide-band type II events}

The details of the SEP analysis for the 25 event dates are listed in Table 4 (online data only). The injection (i.e., first particle release) times with the fixed path length (1.2 AU) method were calculated using the energy channel that showed the earliest onset and the times were corrected by 8.3 min to make the times comparable with electromagnetic observations. The results from the VDA analysis are shown in the table together with comments and the obtained path lengths. The calculated SEP injection times with the VDA method and the corresponding CME height estimates are also shown in Tables 2 and 3. We note that about half of the type II burst events were related with eastern active regions, for which SEP associations are often difficult to establish.

There were 13 events for which SEP injection times could not be calculated with VDA. For five events the SEP onset times could not be determined (labeled NP in Table 2, these five were also all events where the type II burst heights matched those of the CME leading fronts). Seven events occurred during high SEP background and the existence or non-existence of SEPs associated with the type II burst events could not be verified (labeled high bg in Tables 2 and 3, five and two events, respectively). In one event a low-energy precursor led to unphysical VDA results (labeled precursor in Table 2).

Injection times of SEPs could be determined for eleven events (six listed in Table 2 where type II burst heights are in agreement with the CME leading edge heights, five listed in Table 3 where type II heights are significantly less than the CME heights). The CME heights at the time of injection were about zero (three events in Table 2), $\sim 3-4 R_{\odot}$ (three events in Table 2 and two events in Table 3 ), $\sim 8 R_{\odot}$ (one event in Table 3 ), and $\sim 13-26 R_{\odot}$ (two events in Table 3 ). In all events where the CME height at injection time was $\leq 8 R_{\odot}$, the wide-band type II burst 

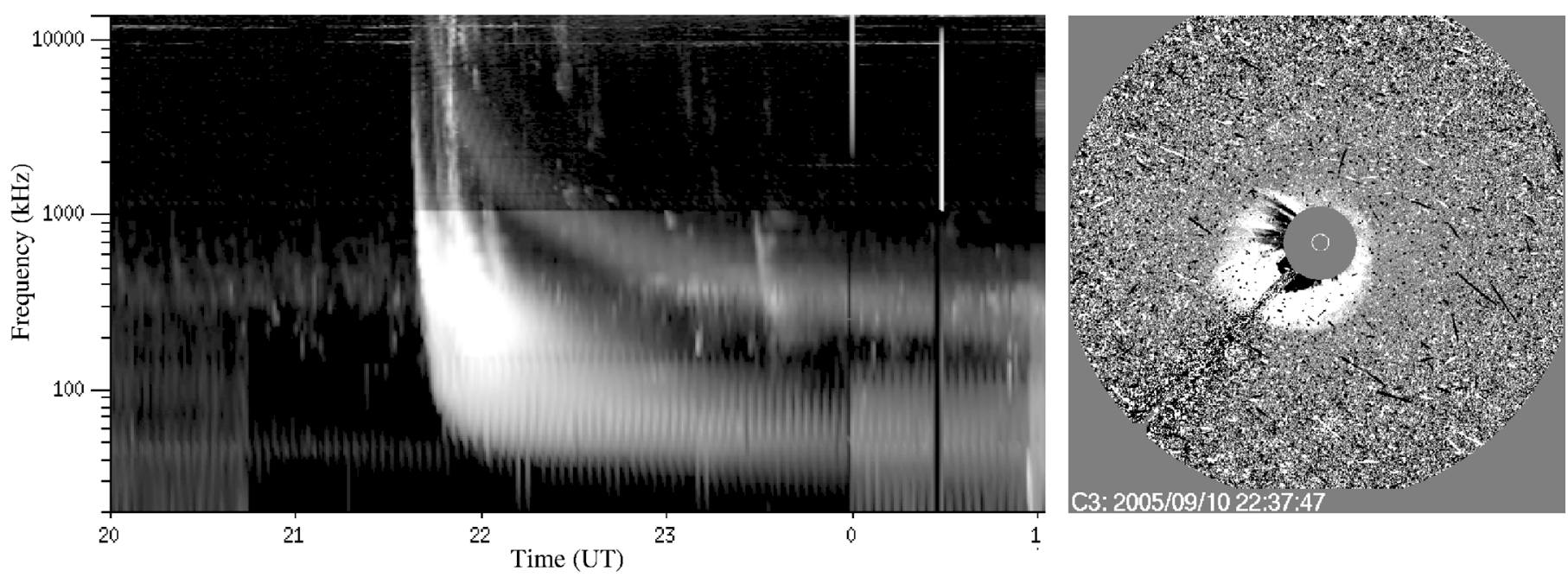

GOES 10 X-Rays:

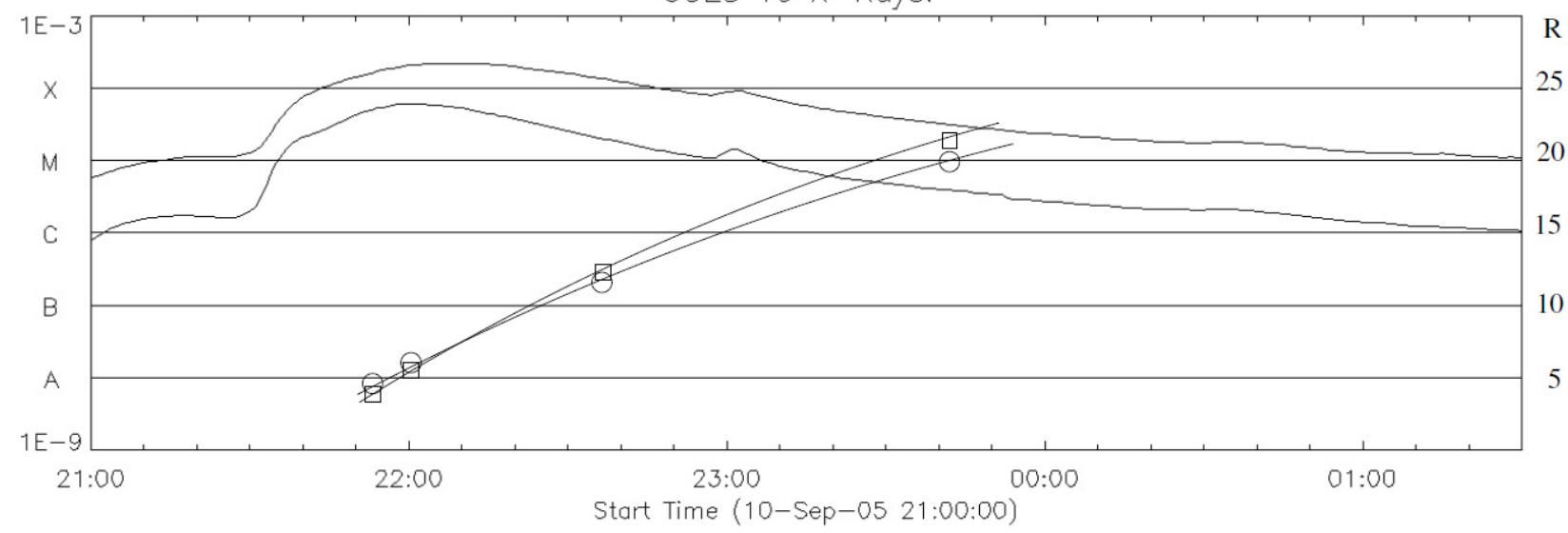

Fig. 27. Wide-band diffuse IP type II burst on 10 September 2005 (event No. 23, top-left panel) observed by the Wind WAVES RAD1 and RAD2 instruments. The LASCO C3 coronagraph image (top-right panel, the small white circle in the center of the image indicates the position of the Sun) shows the CME front at 22:37 UT at height $12.8 R_{\odot}$, at which time the calculated type II burst height was $12.1 R_{\odot}$ (calculated with the hybrid density model, center of the emission lane). This is a rare event where the emission lane is formed above $14 \mathrm{MHz}$ and the lane is fully visible despite the simultaneous strong type III burst activity. GOES plot (bottom panel) shows the flare evolution in soft X-rays. In the same plot the CME and type II heights are indicated with boxes and circles, respectively, with scale shown on the right (heliocentric distance, in solar radii). No SEP injection was identified because of the high particle background.

was observed to appear after the first SEP injection. In most of these before cases the dynamic spectra also showed other emission features like bright patches and separate narrow emission lanes at higher frequencies that could be signatures of acceleration processes, for example, at the flanks of the CMEs (see Figs. 2, 7, 13, 18, 19, and 25). In the two events where SEP acceleration was estimated to start during the wide-band type II emission (Figs. 3 and 4), the dynamic spectra again show additional narrow-band type II and type IV emissions at higher frequencies. Because other simultaneous CMEs were excluded in the event selection process, this suggests that shocks other than CME bow shocks were formed at lower heights, and that they are also possible sources for SEP acceleration.

One event of the 25 seemed clearly to lack SEPs (event No. 10 in Table 2). In this event the type II heights were a match with the CME heights as the type II burst was slightly ahead of the CME leading front, but at start the CME speed $\left(655 \mathrm{~km} \mathrm{~s}^{-1}\right)$ was much less than for the other CMEs. On the other hand, both the CME and the type II burst were accelerating and after the first observation the speeds of both increased significantly $\left(>1000 \mathrm{~km} \mathrm{~s}^{-1}\right)$. Below $1 \mathrm{MHz}$ the wide band type II was very faint. The source region of the eruption was located at S18E15.

\subsection{Preceding events}

In order to compare the CME and type II burst heights, our selection criteria for the data set left out events where multiple CMEs were observed to propagate simultaneously, in the same or different directions. However, preceding CMEs may change the ambient medium and thus affect the densities and plasma frequencies. It has been suggested that preceding CMEs that have large angular widths and originate from the same solar region supply the seed particles for SEP acceleration (e.g., Gopalswamy et al. 2004). To test if a preceding (non-simultaneous) CME with a large angular width $\left(>100^{\circ}\right)$ could be a precondition for the formation of a wide-band type II burst we also calculated the time difference between the launch of a preceding CME and our type II associated CME.

In 16 events a preceding wide CME that originated from the same active region could be identified, and the calculated time differences were two hours (event No. 6), 14-20 h (events No. 5, 13, 18), 24-32 h (events No. 7, 10, 11, 21, 23), approximately two days (events No. 8, 9, 12, 16, 19), and approximately three days (events No. 3, 4). For event No. 15 the first wide preceding CME occurred about three days earlier, but it originated from 

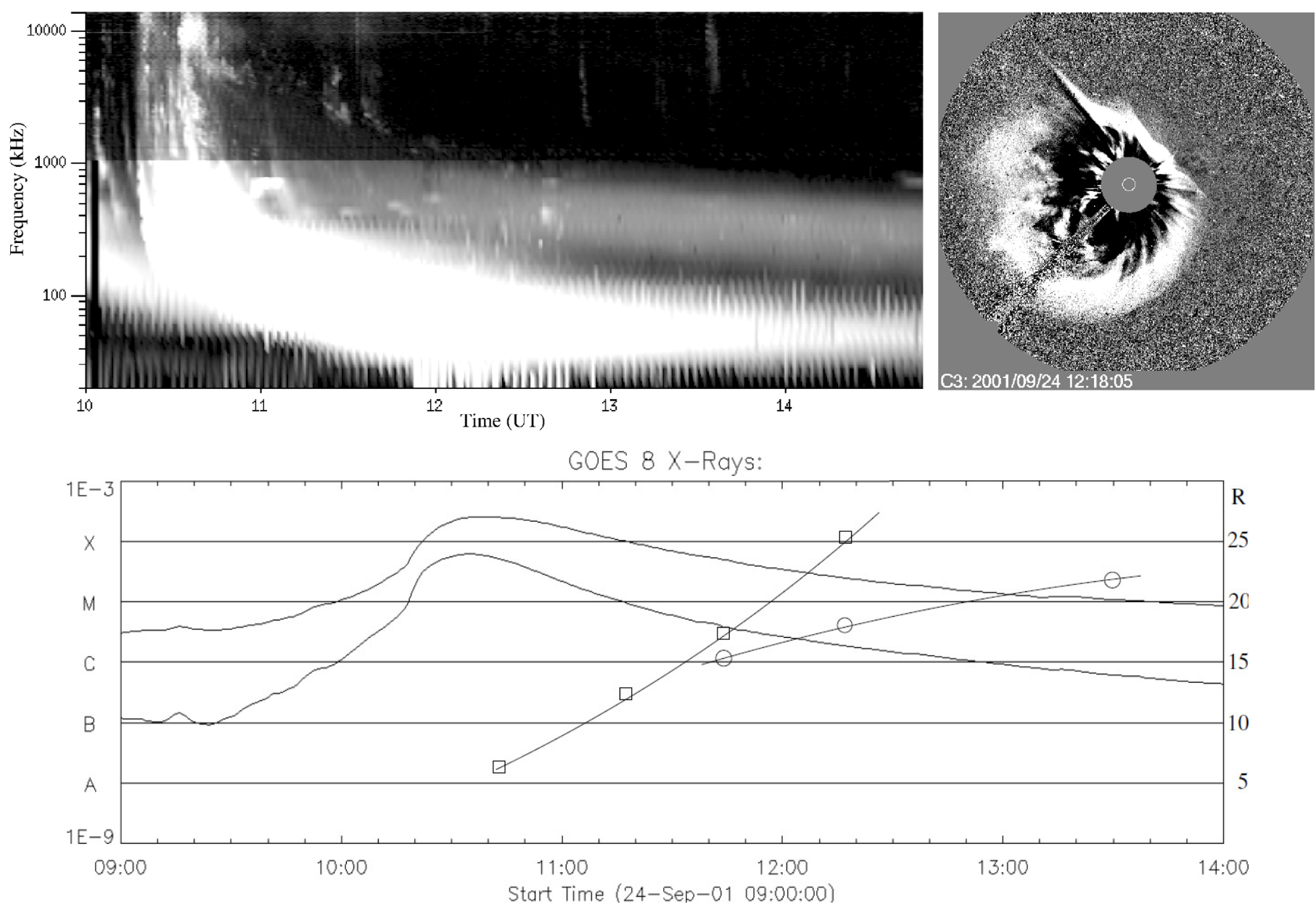

Fig. 28. Wide-band type II event from 24 September 2001 (event No. 1). CME heights are indicated with boxes and type II heights with circles, as in Fig. 27. The type II burst band is mixed with fragmented emission before 11:40 UT and the first type II height is plotted at the time when the full band is visible. The first SEP injection time was calculated to be at 10:31 \pm 8 UT.

another active region. For the other eight limb/backside events the source regions could not be determined, but similar time separations between CMEs were observed. The velocities of the preceding CMEs showed considerable variation, ranging from 250 to $2260 \mathrm{~km} \mathrm{~s}^{-1}$.

\section{Results}

Our basic finding is that two types of diffuse wide-band type II bursts exist: those where the type II burst heights are in agreement with the CME leading edge heights and those where the type II heights are much less and the height difference increases with time. The analyzed 25 wide-band type II bursts were observed between 2001 and 2011, and the radio burst heights were remarkably similar to the CME leading front heights in 18 events. These bursts occurred in 2002-2005, and most of them (14) occurred in 2004-2005, which was during the declining phase of the solar activity cycle and close to the solar minimum. The seven events where the radio burst heights differed from the CME leading front heights occurred in 2001-2003 and 2011, close to the solar activity maximum and during rising phase.

The flares associated with the wide-band IP type II bursts were generally rather intense; there were eight $\mathrm{X}$-class flares, 14 M-class flares, and three C-class flares. Of the three C-class flares, two were partly occulted as they were located at the west limb, and the one on-the-disk C-class flare had C9.6 intensity. Two of the M-class flares may have been occulted as well, as they were located very close to the east limb. Nine of the 25 flares also showed earlier activity (i.e., the flare started during an ongoing earlier flare) or the flares had a double-peak, with two distinctive maximums. The flare and CME source regions were located in various parts of the solar disk: on the eastern hemisphere (10), on the eastern limb (3), on the western hemisphere (8), and on the western limb (4). Eight of the source regions were located near the disk center (within E30-W30).

The associated CMEs had very high speeds (1220$2600 \mathrm{~km} \mathrm{~s}^{-1}$ ), except one (No. 10) where the initial CME speed was only $655 \mathrm{~km} \mathrm{~s}^{-1}$. This was also the event where the SEPs were clearly lacking. The CME speeds were similarly high for events where the type II burst heights matched those of the CME fronts, and for the events where the type II burst heights were much lower. The CME and type II speeds listed in Table 2 show that the height agreement was maintained either when the CME and type II speeds were constant during propagation, or the type II was accelerating and the CME decelerating, or vice versa. In the Table 2 events the calculated type II burst heights were $0.0-4.5 R_{\odot}$ higher than the projected heights of the CME leading fronts at the times of the first measurement, which is in agreement with the CME bow shock scenario.

The speeds listed in Table 3 suggest that the growing difference between the type II and CME heights was either due to 
deceleration in the type II burst velocity or because the type II burst had a much slower velocity from the beginning. In many of the cases the height difference was small in the beginning of the burst, growing significantly larger at larger heights, $>10 R_{\odot}$.

The general appearance of the white-light CMEs associated with the wide-band type II bursts suggests that the shocked regions were wide (the CMEs had wide symmetric fronts) and bow shocks near the CME leading fronts were probable. The large widths of the CME fronts could also explain the wide radio emission bands, since larger sections of the CME (with different atmospheric densities surrounding it) were involved.

We also checked the preceding CMEs, in order to see if earlier CMEs with large angular widths could affect the ambient medium so that the CMEs that were launched later would be able to create wide-band type II bursts. The time difference between the launch of the preceding wide CME and our type II-associated CME turned out to be quite large, from one to two days in most cases. This suggests that earlier events do not play a major role in the formation of wide-band type II emissions, but that the characteristics of the associated CMEs are more important.

During many of the wide-band type II bursts separate burst structures and lanes were also visible at higher frequencies. It is possible that more than one shock appeared simultanously, as shocks may have been formed both at the CME fronts (bow shocks) and at the CME flanks (driven shocks). Since higher frequencies indicate lower atmospheric heights, another shock at the CME flanks is the most plausible explanation if only one propagating CME is observed. Blast wave shocks would be theoretically possible, too, but they are not known to propagate very far from the corona before slowing down and dying out. In one case (event No. 18) the higher-frequency emission lane also looked very wide later on, which may have been a result of the two emissions being observed on top of each other.

Two of the wide-band type II events (events No. 3 and 21) also suggest that the wide-band type II emission may jump to other frequency drift rates. In these two cases the drifts of the emission bands were slightly changed below $1 \mathrm{MHz}$ and $500 \mathrm{kHz}$, respectively (see Table 1 and Figs. 4 and 22). This could be due to non-standard changes in the atmopheric densities. Alternatively, a wide-band burst could also merge with another burst that is created at lower frequencies, but this is very hard to verify because the frequency range is usually not wide enough for both bands to be visible, especially during times of strong type III burst activity.

In terms of SEP association, for only 11 out of the 25 events we were able to determine the first SEP injection times. In seven cases a high background from earlier events prevented the analysis and other uncertanties in the data did the same in six other cases. In one case no SEPs were observed. The estimated CME heights at the time of SEP injection were either near zero (suggesting flare-associated acceleration, three events) or near $\sim 4 R_{\odot}$ (three events) for the events in Table 2. The Table 3 events showed larger distances and/or large uncertainties (two events), or similar intermediate heights of $\sim 3 R_{\odot}$ and $\sim 8 R_{\odot}$ (three events) to those in Table 2. In most of these cases the first SEP injections occurred before the wide-band type II bursts were identified in the dynamic spectra. The dynamic spectra also showed emission features like bright patches and separate narrow emission lanes at higher frequencies, which could be interpreted as signatures of shock waves at heights other than those where the wide-band type II bursts were formed.

In the one event that clearly did not show SEPs (event No. 10), the type II heights and speeds matched those of the CME leading front. In this event the observed CME speed $\left(655 \mathrm{~km} \mathrm{~s}^{-1}\right)$ was significantly lower than in any of the other events and the type II emission was also very weak at frequencies below $1 \mathrm{MHz}$.

\section{Discussion and conclusions}

The suggestion by Bastian (2007) that the wide-band type II emission may not be plasma emission but synchrotron emission from trapped electrons within the CME structure is worth considering. One way to look at the problem is to compare the wide-band type IIs with SEP acceleration, as SEPs may be accelerated by flares or CME-associated shocks. A synchrotron source is not a propagating shock in itself and therefore it would not be capable of accelerating SEPs. However, a fast-moving synchrotron-emitting source would be equally capable of creating shock fronts that could accelerate SEPs. Only in this case could we expect to see more than one type II lane. Another type II burst, with fundamental and harmonic emission bands, was indeed observed during the Bastian event at higher frequencies, which indicates lower burst heights and suggests a shock at the CME flanks.

Our finding that two different types of diffuse wide-band type II bursts exist (those where the type II burst heights suggest they are created by CME bow shocks and those where the bursts seem to be formed somewhere else) could also be explained by the different atmospheric conditions in different phases of solar activity. The densities would affect the type II burst height calculations as most atmospheric models are designed for densities during solar activity minimum.

A propagating shock that creates a diffuse, wide-band type II burst may have characteristics that also affect SEP acceleration. In the cases where the first SEP injection times could be calculated the radio dynamic spectra also showed other features (besides the wide-band emission) that could be interpreted as shock signatures. As the SEP injection time analysis was not possible for all events (some SEP enhancements were too small to be analyzed, some events had energy-dependent precursors that deteriorated the VDA results, and some had high backgrounds from earlier events masking the possible SEP events), no definite conclusions can be made in this respect.

We found evidence of a bow shock origin for most of the wide-band type II events in our sample, but were also able to verify that a separate group of bursts exists where the bow shock scenario does not work. Hence, we cannot exclude the synchrotron source explanation for some of the wide-band type II events.

Acknowledgements. The LASCO CME Catalog is generated and maintained at the CDAW Data Center by NASA and The Catholic University of America in cooperation with the Naval Research Laboratory. SOHO is a project of international cooperation between ESA and NASA. The Wind WAVES instrument was designed and built as a joint effort of the Paris-Meudon Observatory, the University of Minnesota, and the Goddard Space Flight Center, and the data is available at the instrument website. We thank the anonymous referee for valuable comments and suggestions on how to improve the analysis and the presentation of the paper.

\section{References}

Bastian, T. S. 2007, ApJ, 665, 805

Bougeret, J.-L., Kaiser, M. L., Kellogg, P. J., et al. 1995, Space Sci. Rev., 71, 231

Brueckner, G. E., Howard, R. A., Koomen, M. J., et al. 1995, Sol. Phys., 162, 357

Cairns, I. H., Robinson, P. A., \& Zank, G. P. 2000, PASA, 17, 22

Cane, H. V., \& Erickson, W. C. 2005, ApJ, 623, 1180

Cane, H. V., \& Stone, R. G. 1982, NASA-TM-84941, 56 
S. Pohjolainen et al.: Origin of wide-band IP type II bursts

Cane, H. V., Sheeley, N. R., Jr., \& Howard, R. A. 1987, JGR, 92, 9869

Cho, K.-S., Lee, J., Moon, Y.-J., et al. 2007, A\&A, 461, 1121

Gopalswamy, N., Yashiro, S., Kaiser, M. L., Howard, R. A., \& Bougeret, J.-L. 2001, JGR, 106, 29219

Gopalswamy, N., Yashiro, S., Krucker, S., Stenborg, G., \& Howard, R. A. 2004 JGR, 109, A12105

Gopalswamy, N., Yashiro, S., Akiyama, S., et al. 2008a, Ann. Geophys., 26, 3033

Gopalswamy, N., Yashiro, S., Xie, H, et al. 2008b, ApJ, 674, 560

Hoang, S., Pantellini, F., Harvey, C. C., et al. 1992, in Solar Wind Seven Colloquium, eds. E. Marsch, \& R. Schwenn, 465

Huttunen-Heikinmaa, K., Valtonen, E., \& Laitinen, T. 2005, A\&A, 442, 673

Kahler, S., \& Ragot, B. R. 2006, ApJ, 646, 634

Kim, Yeon-Han, Bong, Su-Chan, Park, Y.-D., Cho, K.-S., \& Moon, Y.-J. 2009, ApJ, 705, 1721

Leblanc, Y., Dulk, G. A., \& Bougeret, J.-L. 1998, Sol. Phys., 183, 165
Lintunen, J., \& Vainio, R. 2004, A\&A, 420, 343

Mann, G., Classen, T., \& Aurass, H. 1995, A\&A, 295, 775

Melrose, D. B. 1980, Space. Sci. Rev., 26, 3

Pohjolainen, S., van Driel-Gesztelyi, L., Culhane, J. L., Manoharan, P. K., \& Elliott, H. A. 2007, Sol. Phys., 244, 167

Saito, K. 1970, Ann. Tokyo Astron. Obs., 12, 53

Torsti, J., Valtonen, E., Lumme, M., et al. 1995, Sol. Phys., 162, 505

Vourlidas, A., Pick, M., Hoang, S., \& Demoulin, P. 2007a, ApJ, 656, L105

Vourlidas, A., Pick, M., Hoang, S., \& Demoulin, P. 2007b, ApJ, 665, L179

Vrsnak, B., Aurass, H., Magdalenic, J., \& Gopalswamy, N. 2001, A\&A, 377, 321

Vrsnak, B., Magdalenic, J., \& Zlobec, P. 2004, A\&A, 413, 753

Vrsnak, B., Warmuth, A., \& Temmer, M. 2006, A\&A, 448, 739

Warmuth, A. 2007, in The High Energy Solar Corona: Waves, Eruptions, Particles, eds. K.-L. Klein, \& A. MacKinnon (Berlin Heidelberg: Springer Verlag), Lect. Notes Phys., 725, 107 
A\&A 558, A7 (2013)

\begin{tabular}{|c|c|c|c|c|c|c|}
\hline 1 & & & & & & \\
\hline Time (UT) & $10: 31$ & $10: 42$ & $11: 18$ & $11: 42$ & $12: 18$ & $13: 30$ \\
\hline CME height $\left(R_{\odot}\right)$ & 3.3 & 5.2 & 12.7 & 17.5 & 25.4 & \\
\hline type II height $\left(R_{\odot}\right)$, hybrid model & & & & 15.6 & 18.0 & 22.0 \\
\hline type II frequency $(\mathrm{kHz})$, lane center & & & & 500 & 400 & 300 \\
\hline
\end{tabular}
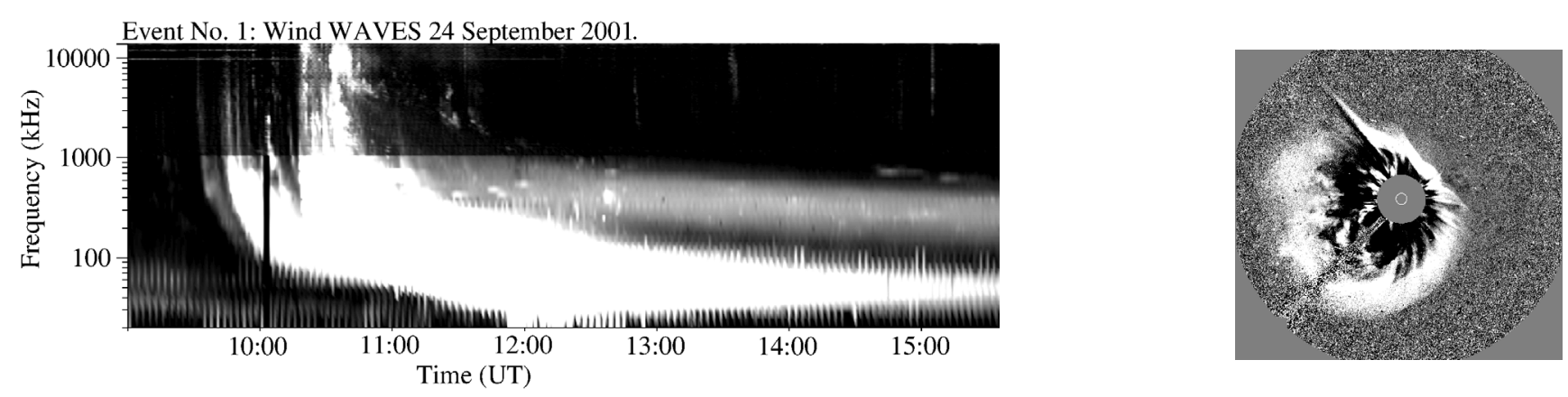

Fig. 2. Estimated CME and type II burst heights for the 24 September 2001 event (Event No. 1). The Wind WAVES radio dynamic spectrum is plotted in the frequency range $20-13825 \mathrm{kHz}$. The spectrum is measured by two instruments, RAD1 and RAD2, and the change in instrument sensitivity is visible near $1000 \mathrm{kHz}$. The LASCO C3 difference image shows the CME at 12:18 UT, at height $25.4 R_{\odot}$, at central position angle of $141^{\circ}$ (counterclockwise from the top). This was a full halo CME. Estimated first SEP injection time was at 10:31 \pm 8 UT.

\begin{tabular}{|c|c|c|c|c|c|c|c|c|c|}
\hline vent No. 2 & & & & & & & & & \\
\hline Time (UT) & $05: 35$ & $07: 15$ & $07: 30$ & $07: 45$ & 08:00 & $08: 15$ & $08: 30$ & $08: 45$ & $10: 00$ \\
\hline CME height $\left(R_{\odot}\right)$ & 2.7 & 14.0 & 16.1 & 18.3 & 19.8 & 21.9 & 23.8 & 26.2 & \\
\hline type II height $\left(R_{\odot}\right)$, h & & & 13. & 15.4 & 16. & 17.5 & 18.0 & 19.7 & 25.0 \\
\hline type II frequency $(\mathrm{kHz})$, lane center & & & 650 & 510 & 460 & 420 & 400 & 350 & 250 \\
\hline
\end{tabular}
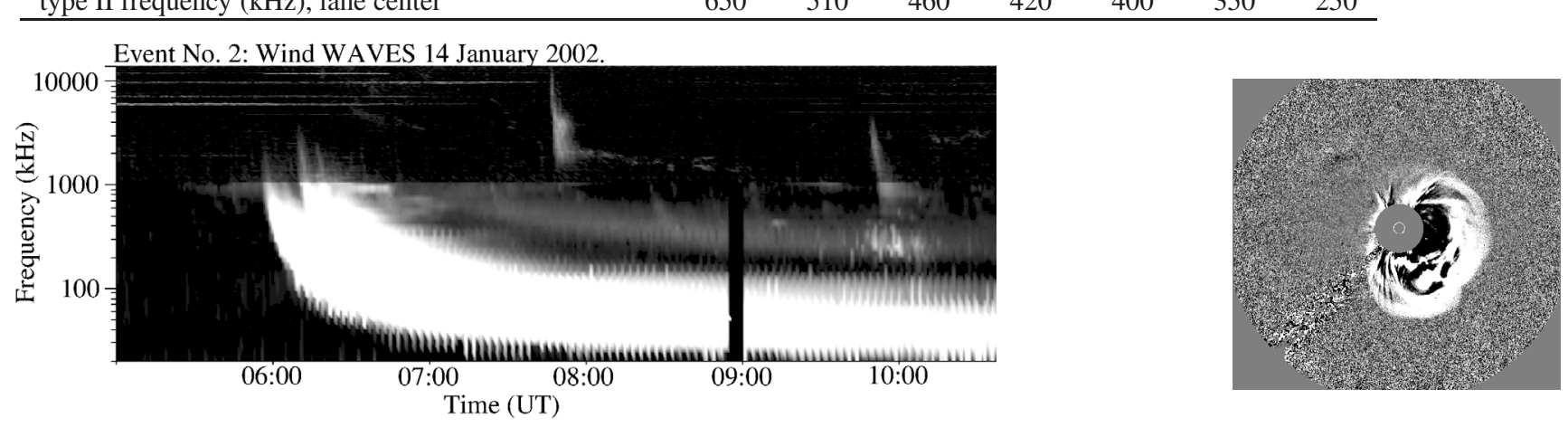

Fig. 3. Estimated CME and type II burst heights for the 14 January 2002 event (Event No. 2). The Wind WAVES frequency range is as in Fig. 2. The LASCO C2 image difference image shows the CME at 07:30 UT, at height $16.1 R_{\odot}$, at central position angle of $243^{\circ}$ (counterclockwise from the top). This was a full halo CME. Estimated first SEP injection time was at 7:56 $\pm 48 \mathrm{UT}$.

\begin{tabular}{|c|c|c|c|c|c|c|c|}
\hline \\
\hline Time (UT) & $12: 30$ & $12: 42$ & $12: 54$ & $13: 42$ & $14: 18$ & $14: 42$ & $15: 18$ \\
\hline $\mathrm{CME}$ height $\left(R_{\odot}\right)$ & 3.7 & 5.5 & 6.9 & 14.4 & 19.1 & 22.5 & 26.0 \\
\hline type II height $\left(R_{\odot}\right)$, hybrid model & 4.9 & 5.9 & 6.9 & 11.1 & & 12.6 & \\
\hline type II frequency $(\mathrm{kHz})$, lane center & 4200 & 2800 & 2100 & 880 & & 710 & \\
\hline
\end{tabular}
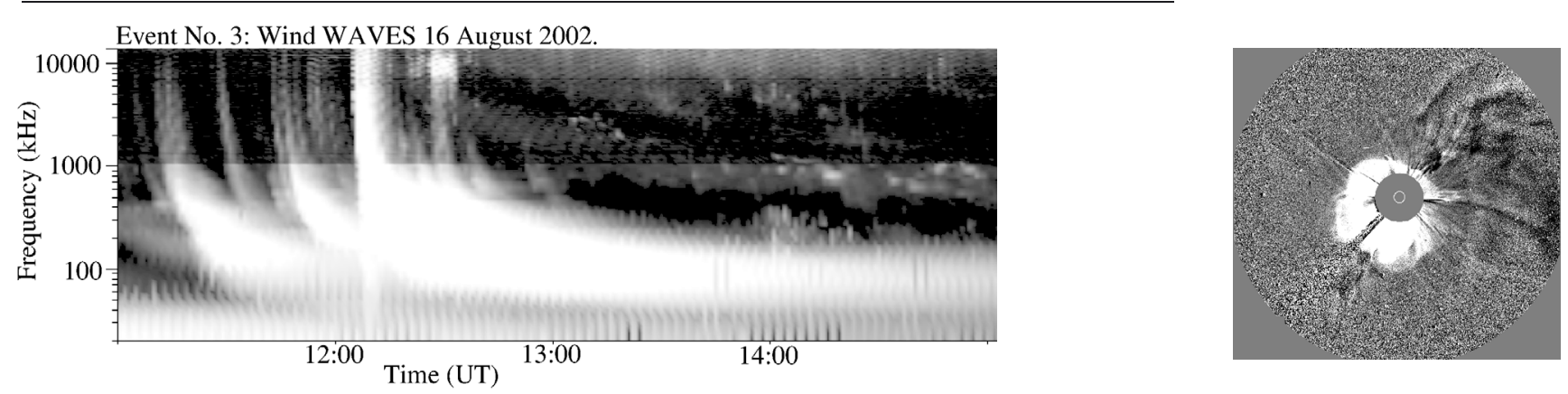

Fig. 4. Estimated CME and type II burst heights for the 16 August 2002 event (Event No. 3). The Wind WAVES frequency range is as in Fig. 2. The LASCO C2 image difference image shows the CME at 13:42 UT, at height $14.4 R_{\odot}$, at central position angle of $121^{\circ}$ (counterclockwise from the top). This was a full halo CME. Estimated first SEP injection time was at 14:20 \pm 11 UT. 
S. Pohjolainen et al.: Origin of wide-band IP type II bursts

\begin{tabular}{|c|c|c|c|c|c|c|}
\hline \multicolumn{7}{|l|}{ Event No. 4} \\
\hline Time (UT) & $13: 31$ & $13: 42$ & $14: 18$ & $14: 42$ & $15: 18$ & $15: 42$ \\
\hline $\mathrm{CME}$ height $\left(R_{\odot}\right)$ & 4.2 & 5.9 & 11.1 & 14.8 & 21.2 & 24.7 \\
\hline type II height $\left(R_{\odot}\right)$, hybrid model & 4.4 & 6.6 & 12.6 & & & \\
\hline type II frequency $(\mathrm{kHz})$, lane center & 5100 & 2300 & 700 & & & \\
\hline
\end{tabular}
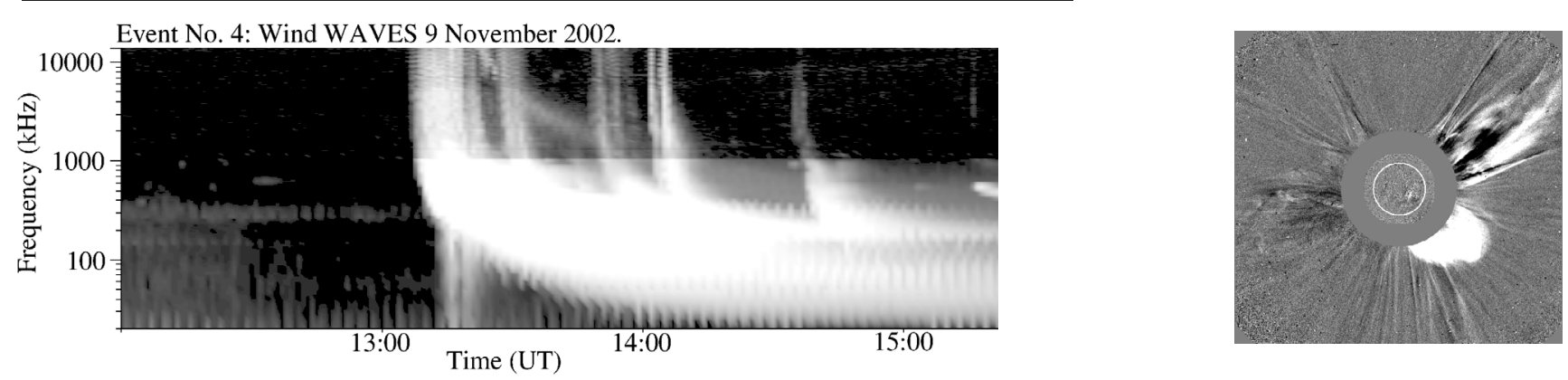

Fig. 5. Estimated CME and type II burst heights for the 9 November 2002 event (Event No. 4). The Wind WAVES frequency range is as in Fig. 2. The LASCO C2 image difference image shows the CME at 13:31 UT, at height $4.2 R_{\odot}$, at central position angle of $234^{\circ}$ (counterclockwise from the top). This was a full halo CME. Estimated first SEP injection time was at 13:05 \pm 9 UT.

\begin{tabular}{lrrrr} 
Event No. 5 & & & & \\
\hline Time (UT) & $03: 30$ & $03: 42$ & $04: 18$ & $05: 18$ \\
CME height $\left(R_{\odot}\right)$ & 4.7 & 6.9 & 12.1 & 20.4 \\
type II height $\left(R_{\odot}\right)$, hybrid model & & 7.1 & 11.7 & \\
type II frequency $(\mathrm{kHz})$, lane center & & 2000 & 800 & \\
\hline
\end{tabular}

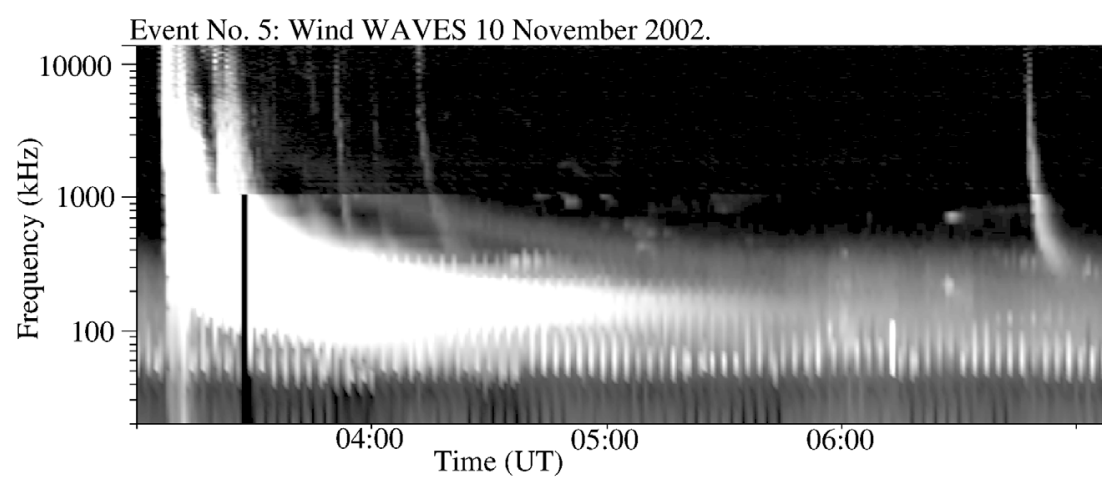

Fig. 6. Estimated CME and type II burst heights for the 10 November 2002 event (Event No. 5). The Wind WAVES frequency range is as in Fig. 2. The LASCO C2 image difference image shows the CME at 04:18 UT, at height $12.1 R_{\odot}$, at central position angle of $228^{\circ}$ (counterclockwise from the top). This CME had a width of $282^{\circ}$. SEP injection time analysis was not possible.

\begin{tabular}{|c|c|c|c|c|c|c|c|}
\hline \multicolumn{8}{|l|}{ Event No. 6} \\
\hline Time (UT) & $00: 50$ & $01: 42$ & $02: 18$ & $02: 42$ & $03: 18$ & $03: 42$ & $04: 18$ \\
\hline CME height $\left(R_{\odot}\right)$ & 5.0 & 9.9 & 14.1 & 17.6 & 21.7 & 24.5 & 29.2 \\
\hline type II height $\left(R_{\odot}\right)$, hybrid model & & 14.0 & 18.0 & 21.0 & 22.0 & & \\
\hline type II frequency $(\mathrm{kHz})$, lane center & & 600 & 400 & 320 & 300 & & \\
\hline
\end{tabular}

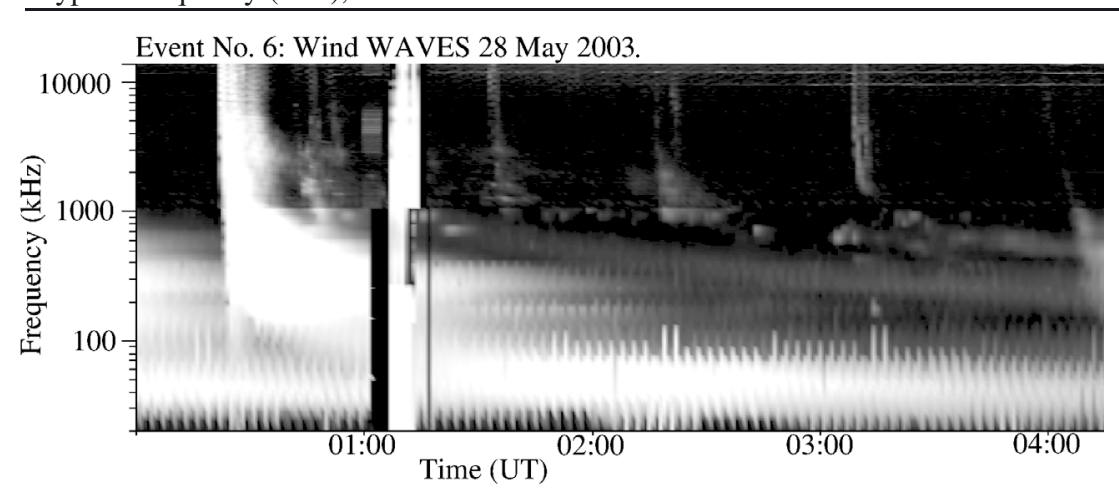

Fig. 7. Estimated CME and type II burst heights (lower diffuse lane) for the 28 May 2003 event (Event No. 6). The Wind WAVES frequency range as in Fig. 2. The LASCO C2 image difference image shows the CME at 02:18 UT, at height $14.1 R_{\odot}$, at central position angle of $293^{\circ}$ (counterclockwise from the top). This was a full halo CME, preceded by another fast halo CME one hour earlier. Estimated first SEP injection time was at 00:15 \pm 11 UT. 


\begin{tabular}{|c|c|c|c|c|c|c|c|}
\hline & & & & & & & \\
\hline Time (UT) & $01: 16$ & $01: 27$ & 01:42 & $02: 18$ & $03: 18$ & $04: 18$ & $04: 42$ \\
\hline CME height $\left(R_{\odot}\right)$ & & 5.0 & 6.9 & 11.3 & 17.1 & 23.4 & 25.9 \\
\hline type II height $\left(R_{\odot}\right)$, hybrid model & 4.5 & 6.6 & 7.7 & 12.6 & & & \\
\hline type II frequency $(\mathrm{kHz})$, lane center & 4800 & 2300 & 1700 & 700 & & & \\
\hline
\end{tabular}
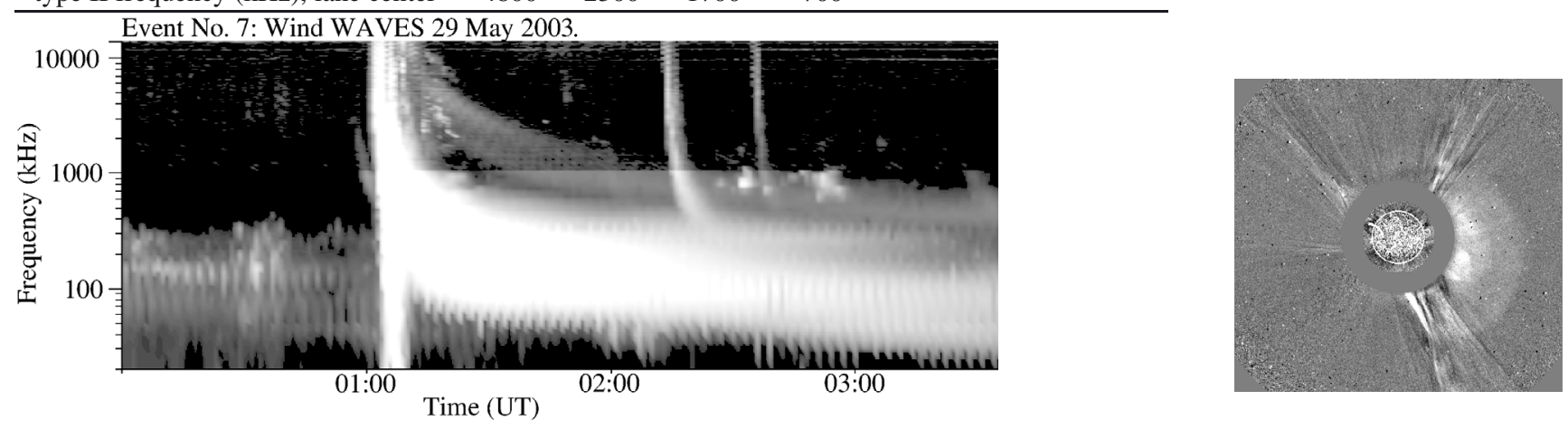

Fig. 8. Estimated CME and type II burst heights for the 29 May 2003 event (Event No. 7). The Wind WAVES frequency range is as in Fig. 2. The LASCO C2 image difference image shows the CME at 01:27 UT, at height $4.9 R_{\odot}$, at central position angle of $262^{\circ}$ (counterclockwise from the top). This was a full halo CME. SEP injection time analysis was not possible.
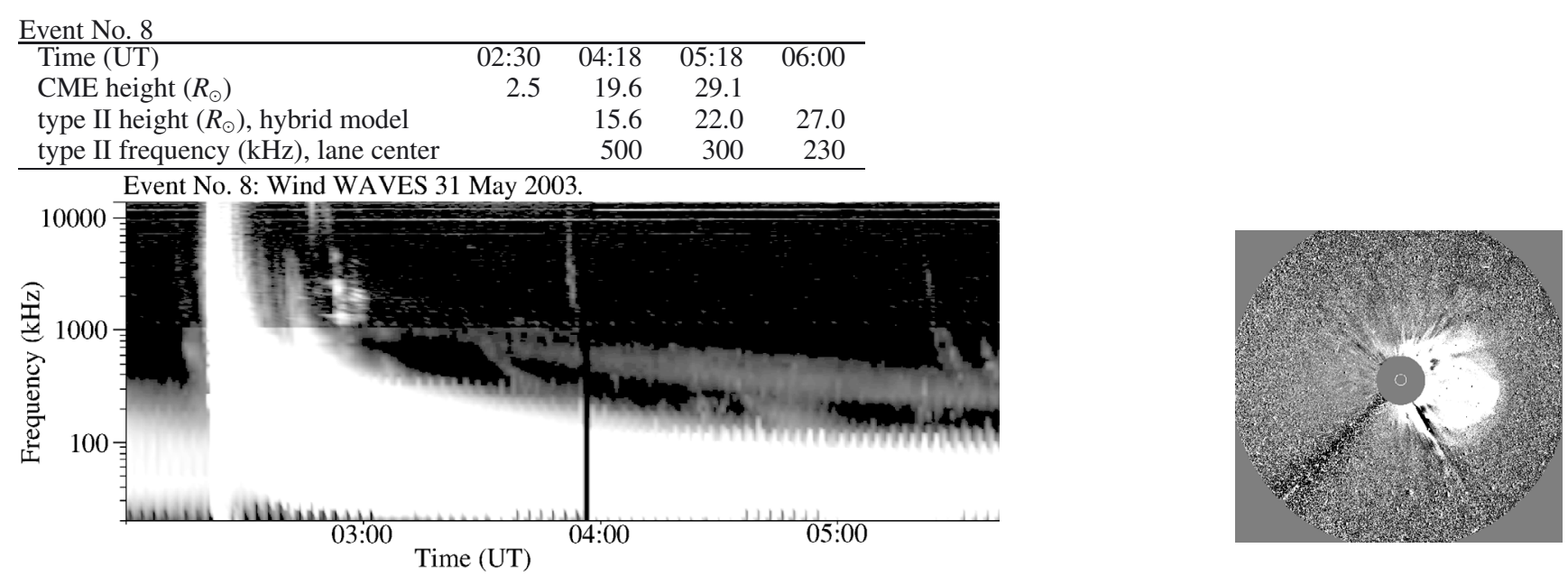

Fig. 9. Estimated CME and type II burst heights for the 31 May 2003 event (Event No. 8). The Wind WAVES frequency range is as in Fig. 2. The LASCO C2 image difference image shows the CME at 04:18 UT, at height $19.6 R_{\odot}$, at central position angle of $255^{\circ}$ (counterclockwise from the top). This was a full halo CME. Estimated first SEP injection time was at 02:30 \pm 4 UT.
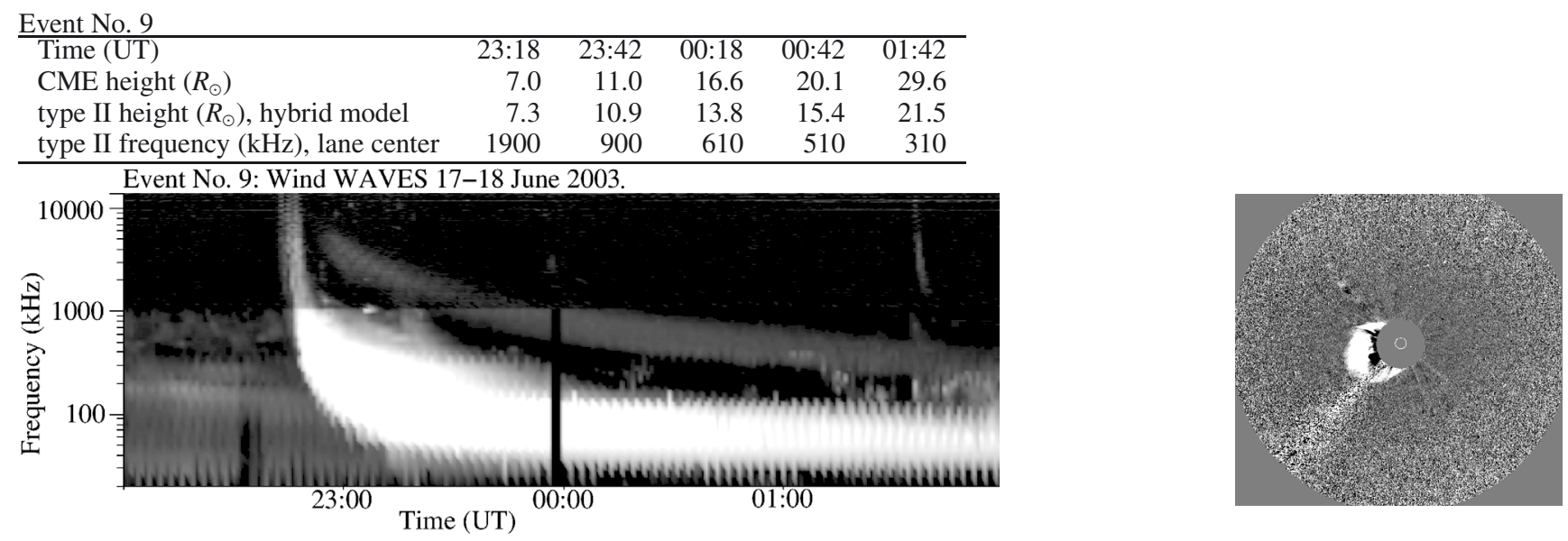

Fig. 10. Estimated CME and type II burst heights for the 17-18 June 2003 event (Event No. 9). The Wind WAVES frequency range is as in Fig. 2. The LASCO C2 image difference image shows the CME at 23:42 UT, at height $11.0 R_{\odot}$, at central position angle of $117^{\circ}$ (counterclockwise from the top). This was a full halo CME. SEP injection time analysis was not possible. 
S. Pohjolainen et al.: Origin of wide-band IP type II bursts

\begin{tabular}{|c|c|c|c|c|c|c|c|}
\hline Event No. 10 & & & & & & & \\
\hline Time (UT) & $13: 31$ & $13: 54$ & $14: 06$ & $14: 18$ & $15: 18$ & $16: 18$ & $16: 42$ \\
\hline CME height $\left(R_{\odot}\right)$ & 3.9 & 5.2 & 6.3 & 8.0 & 15.2 & 22.4 & 25.6 \\
\hline type II height $\left(R_{\odot}\right)$, hybrid model & 4.5 & 6.4 & 7.5 & & & & \\
\hline type II frequency $(\mathrm{kHz})$, lane center & 4800 & 2400 & 1800 & & & & \\
\hline
\end{tabular}
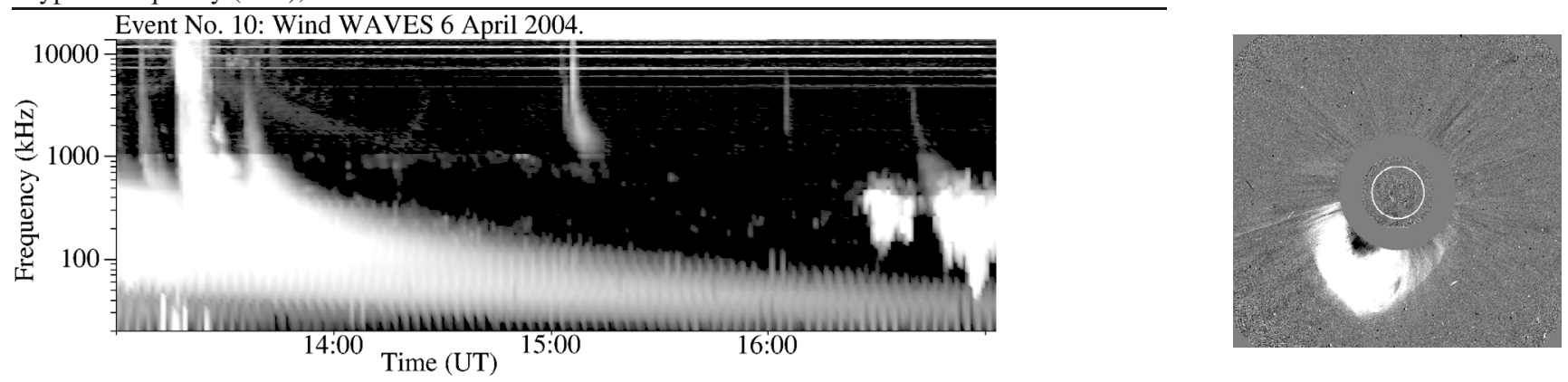

Fig. 11. Estimated CME and type II burst heights for the 6 April 2004 event (Event No. 10). The Wind WAVES frequency range is as in Fig. 2. The LASCO C2 image difference image shows the CME at 13:54 UT, at height 5.2 $R_{\odot}$, at central position angle of $164^{\circ}$ (counterclockwise from the top). This was a full halo CME. No SEPs were observed.
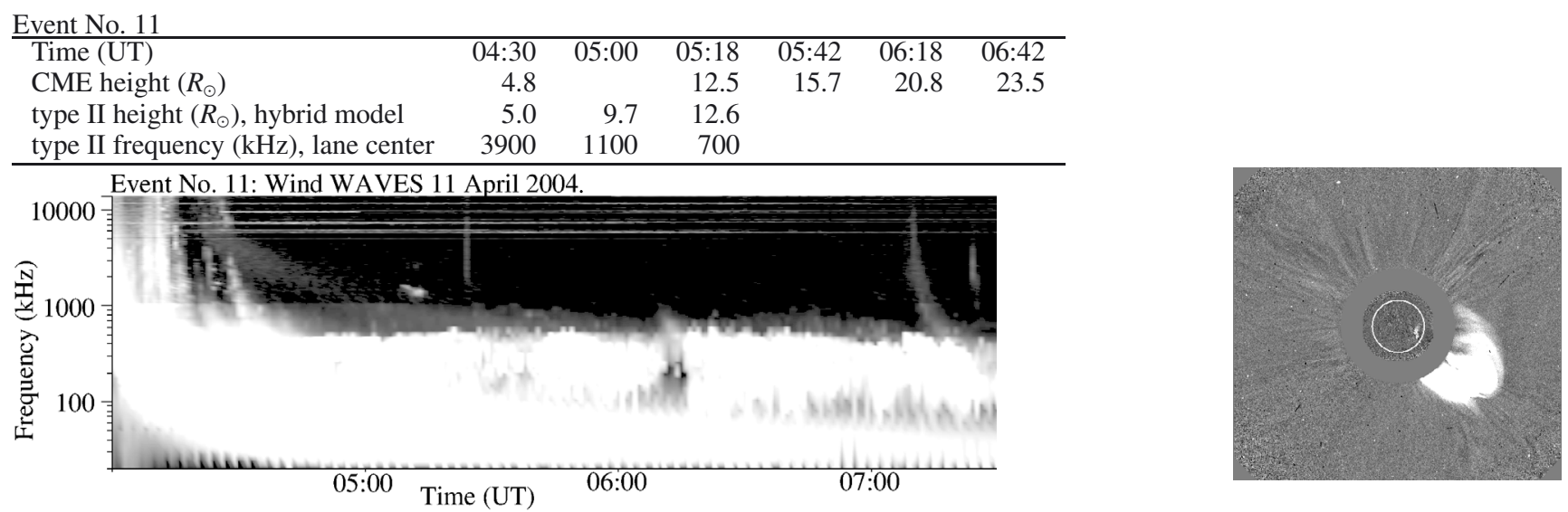

Fig. 12. Estimated CME and type II burst heights for the 11 April 2004 event (Event No. 11). The Wind WAVES frequency range is as in Fig. 2. The LASCO C2 image difference image shows the CME at 04:30 UT, at height $4.8 R_{\odot}$, at central position angle of $237^{\circ}$ (counterclockwise from the top). This CME had a width of $314^{\circ}$. Estimated first SEP injection time was at 04:06 \pm 6 UT.

\begin{tabular}{|c|c|c|c|c|c|c|c|c|c|}
\hline 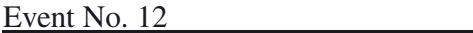 & & & & & & & & & \\
\hline Time (UT) & $14: 30$ & $14: 54$ & $15: 06$ & $15: 18$ & $15: 30$ & $15: 42$ & $16: 18$ & $17: 18$ & $17: 42$ \\
\hline $\mathrm{CME}$ height $\left(R_{\odot}\right)$ & 3.2 & 4.1 & 4.2 & 5.2 & 6.4 & 8.0 & 12.1 & 19.0 & 21.9 \\
\hline type II height $\left(R_{\odot}\right)$, hybrid model & & & & & 10.9 & 12.1 & 15.2 & 22.0 & 25.0 \\
\hline type II frequency $(\mathrm{kHz})$, lane center & & & & & 900 & 750 & 520 & 300 & 250 \\
\hline
\end{tabular}

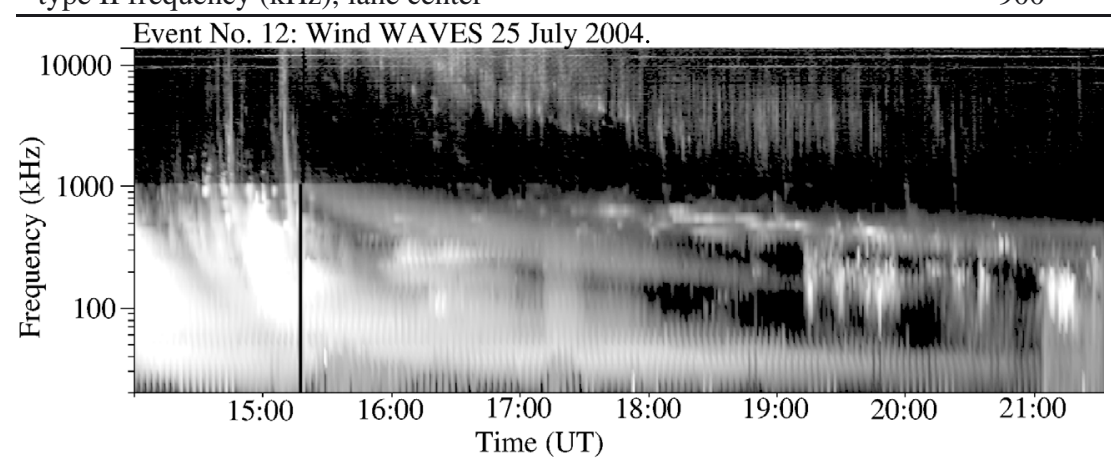

Fig. 13. Estimated CME and type II burst heights (lower diffuse lane) for the 25 July 2004 event (Event No. 12). The Wind WAVES frequency range is as in Fig. 2. The LASCO C3 image difference image shows the CME at 16:18 UT, at height $12.1 R_{\odot}$, at central position angle of $204^{\circ}$ (counterclockwise from the top). This was a full halo CME. Estimated first SEP injection time was at 15:04 \pm 6 UT. 
A\&A 558, A7 (2013)

\begin{tabular}{|c|c|c|c|c|c|c|c|c|c|}
\hline Event No. 13 & & & & & & & & & \\
\hline Time (UT) & $12: 06$ & $12: 30$ & $12: 54$ & $13: 36$ & $14: 18$ & $14: 42$ & $15: 23$ & $15: 44$ & $16: 18$ \\
\hline CME height $\left(R_{\odot}\right)$ & 3.1 & 3.8 & 4.9 & & 14.3 & 17.2 & 21.5 & 24.2 & 27.5 \\
\hline type II height $\left(R_{\odot}\right)$, hybrid model & & & & 12.6 & 16.5 & 18.6 & 22.0 & 23.1 & \\
\hline type II frequency $(\mathrm{kHz})$, lane center & & & & 700 & 460 & 390 & 300 & 280 & \\
\hline
\end{tabular}

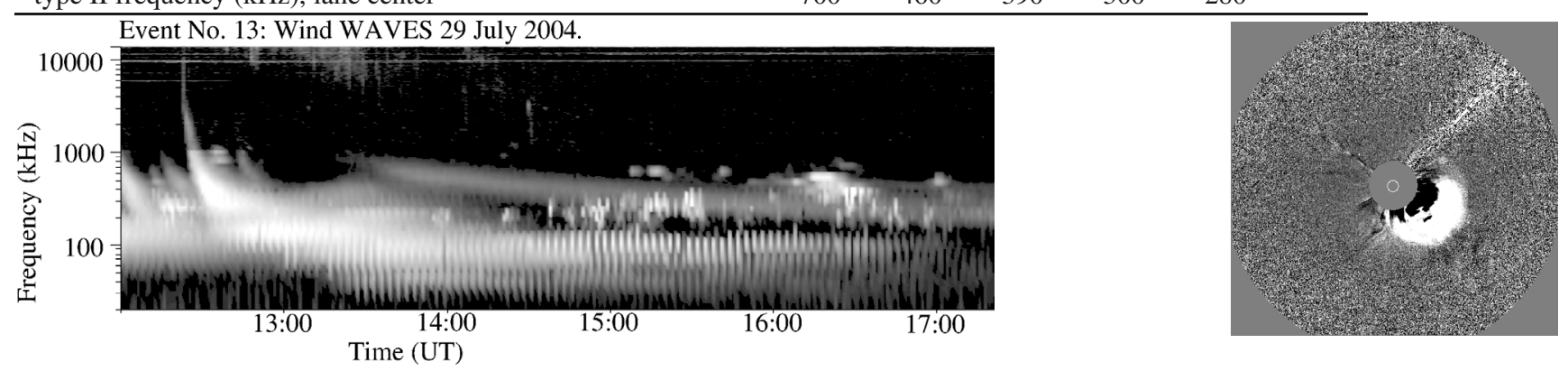

Fig. 14. Estimated CME and type II burst heights for the 29 July 2004 event (Event No. 13). The Wind WAVES frequency range is as in Fig. 2. The LASCO C3 image difference image shows the CME at 14:18 UT, at height $14.3 R_{\odot}$, at central position angle of $243^{\circ}$ (counterclockwise from the top). This was a full halo CME. SEP injection time analysis was not possible.
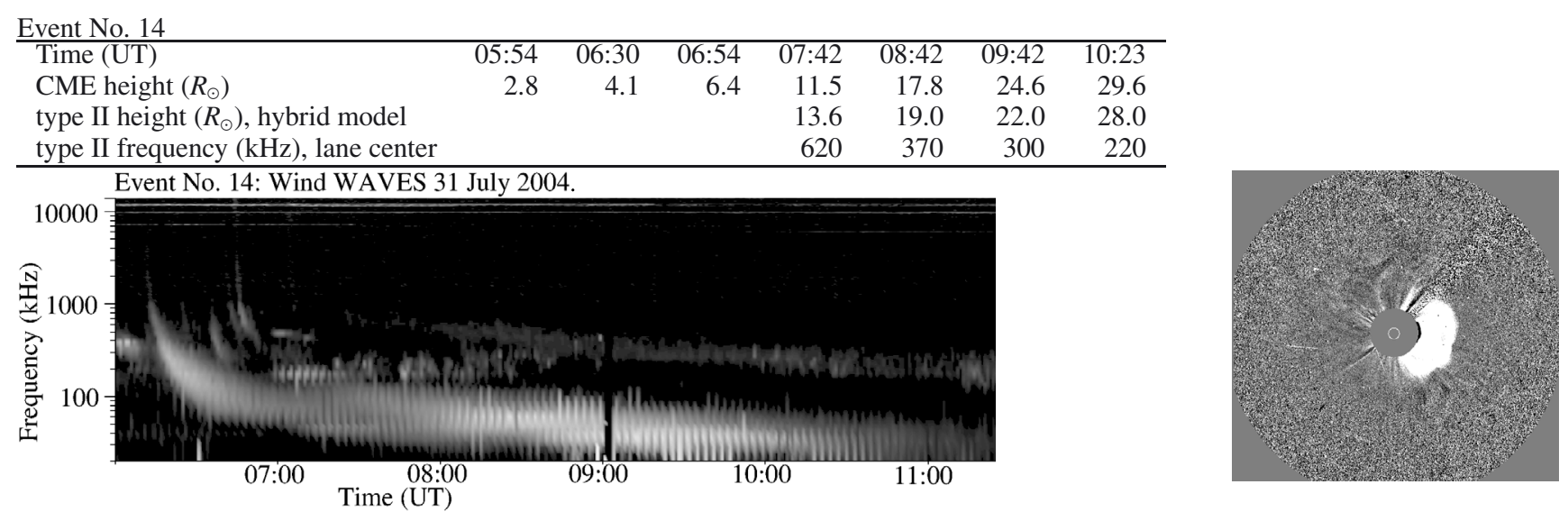

Fig. 15. Estimated CME and type II burst heights for the 31 July 2004 event (Event No. 14). The Wind WAVES frequency range is as in Fig. 2. The LASCO C3 image difference image shows the CME at 07:42 UT, at height $11.5 R_{\odot}$, at central position angle of $287^{\circ}$ (counterclockwise from the top). This CME had a width of $>197^{\circ}$. SEP injection time analysis was not possible.

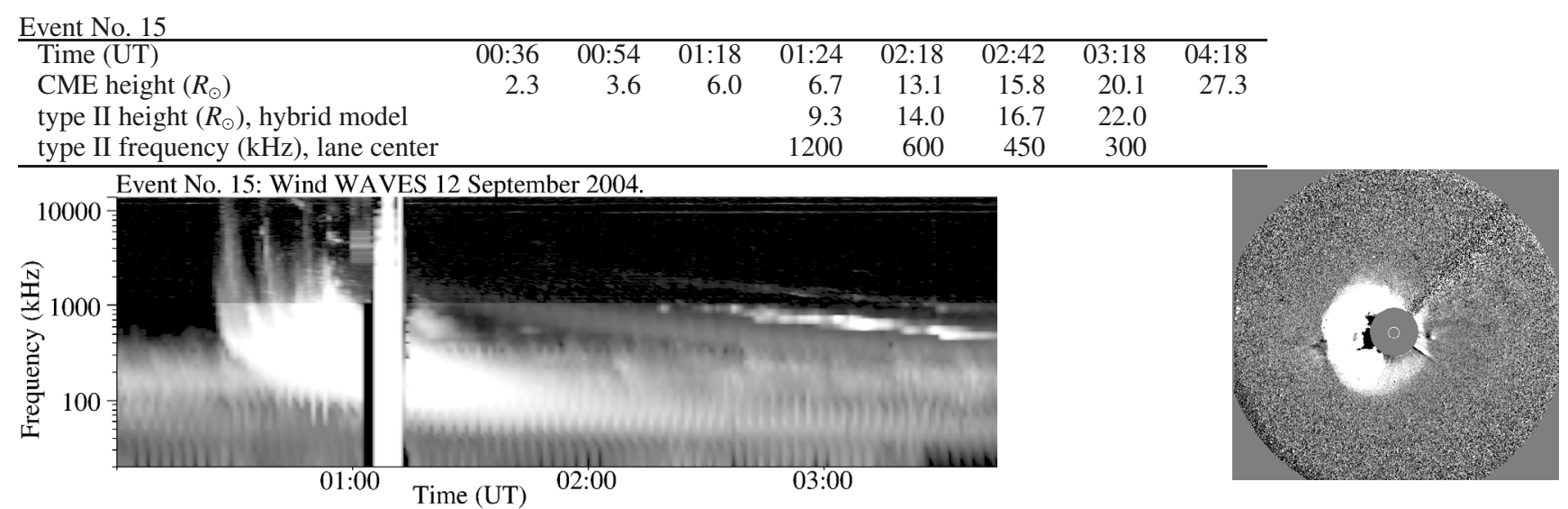

Fig. 16. Estimated CME and type II burst heights (lower diffuse lane) for the 12 September 2004 event (Event No. 15). The Wind WAVES frequency range is as in Fig. 2. The LASCO C3 image difference image shows the CME at 02:18 UT, at height $13.1 R_{\odot}$, at central position angle of $133^{\circ}$ (counterclockwise from the top). This was a full halo CME. SEP injection time analysis was not possible. 
S. Pohjolainen et al.: Origin of wide-band IP type II bursts

\begin{tabular}{|c|c|c|c|c|c|c|c|}
\hline Event No. 16 & & & & & & & \\
\hline Time (UT) & $00: 26$ & $00: 42$ & $00: 50$ & $01: 42$ & $02: 18$ & $02: 42$ & $03: 18$ \\
\hline CME height $\left(R_{\odot}\right)$ & 4.2 & 5.8 & 7.0 & 12.6 & 16.3 & 18.4 & 22.2 \\
\hline type II height $\left(R_{\odot}\right)$, hybrid model & 6.1 & & 9.3 & 14.0 & & & \\
\hline type II frequency $(\mathrm{kHz})$, lane center & 2700 & & 1200 & 600 & & & \\
\hline
\end{tabular}
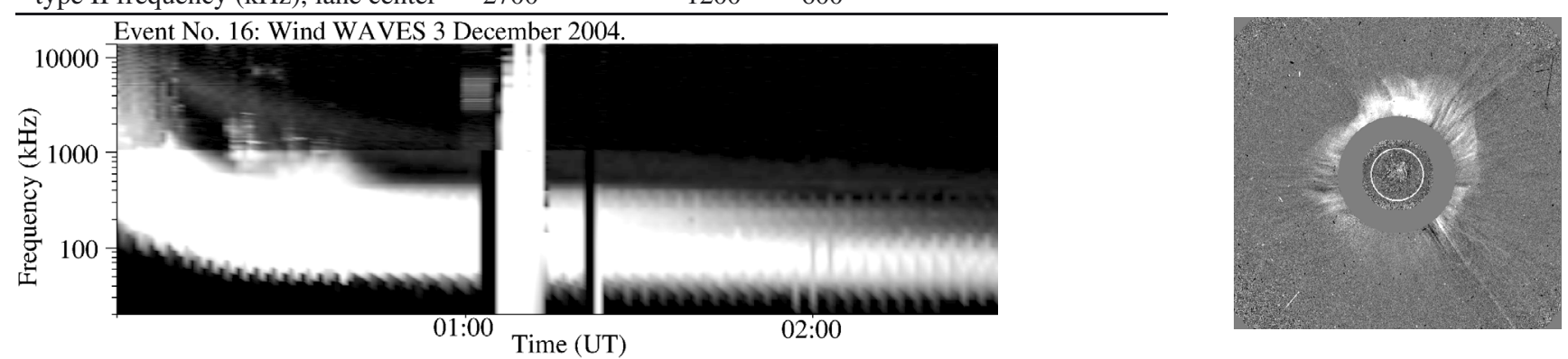

Fig. 17. Estimated CME and type II burst heights for the 3 December 2004 event (Event No. 16). The Wind WAVES frequency range is as in Fig. 2. The LASCO C2 image difference image shows the CME at 00:26 UT, at height $4.2 R_{\odot}$, at central position angle of $328^{\circ}$ (counterclockwise from the top). This was a full halo CME. SEP injection time analysis was not possible.

\begin{tabular}{|c|c|c|c|c|c|}
\hline Event No. 17 & & & & & \\
\hline Time (UT) & $06: 30$ & $06: 42$ & $07: 42$ & 08:42 & 09:00 \\
\hline CME height $\left(R_{\odot}\right)$ & 5.8 & 7.9 & 18.8 & 29.0 & \\
\hline type II height $\left(R_{\odot}\right)$, hy & & 11 & 21.0 & 28.0 & 29.0 \\
\hline type II frequency $(\mathrm{kHz})$, lane center & & 800 & 320 & 220 & 210 \\
\hline
\end{tabular}
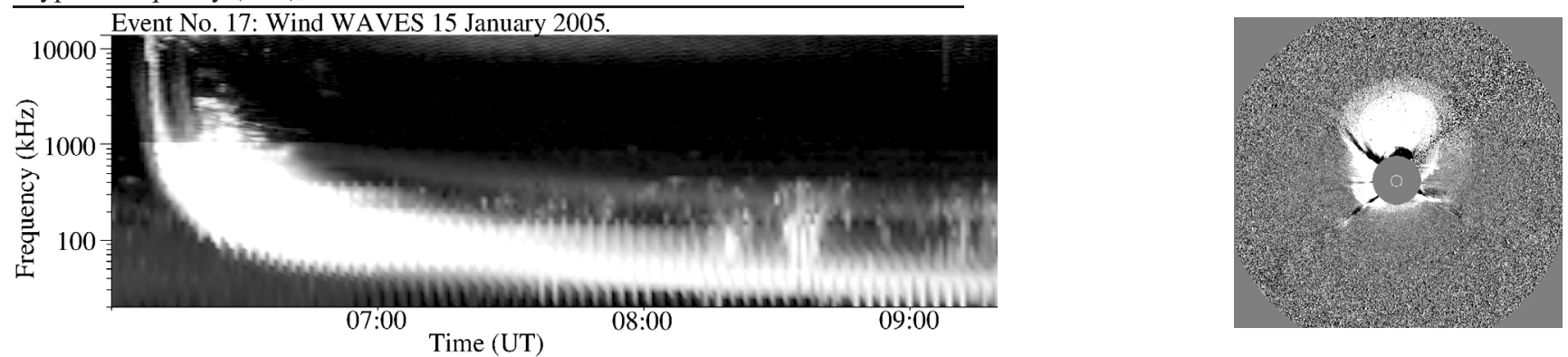

Fig. 18. Estimated CME and type II burst heights for the 15 January 2005 event (Event No. 17). The Wind WAVES frequency range is as in Fig. 2. The LASCO C3 image difference image shows the CME at 07:42 UT, at height $18.8 R_{\odot}$, at central position angle of $358^{\circ}$ (counterclockwise from the top). This was a full halo CME. Estimated first SEP injection time was at 06:20 \pm 4 UT.

\begin{tabular}{|c|c|c|c|c|c|}
\hline 18 & & & & & \\
\hline Time (UT) & 23:06 & $23: 18$ & $23: 42$ & $00: 18$ & 01:00 \\
\hline $\mathrm{CME}$ height $\left(R_{\odot}\right)$ & 7.3 & 10.4 & 16.5 & 24.9 & \\
\hline type II height $\left(R_{\odot}\right)$, hybrid model & & & 16.5 & 23.0 & 9.0 \\
\hline type II frequency $(\mathrm{kHz})$, lane center & & & 460 & 280 & 210 \\
\hline
\end{tabular}
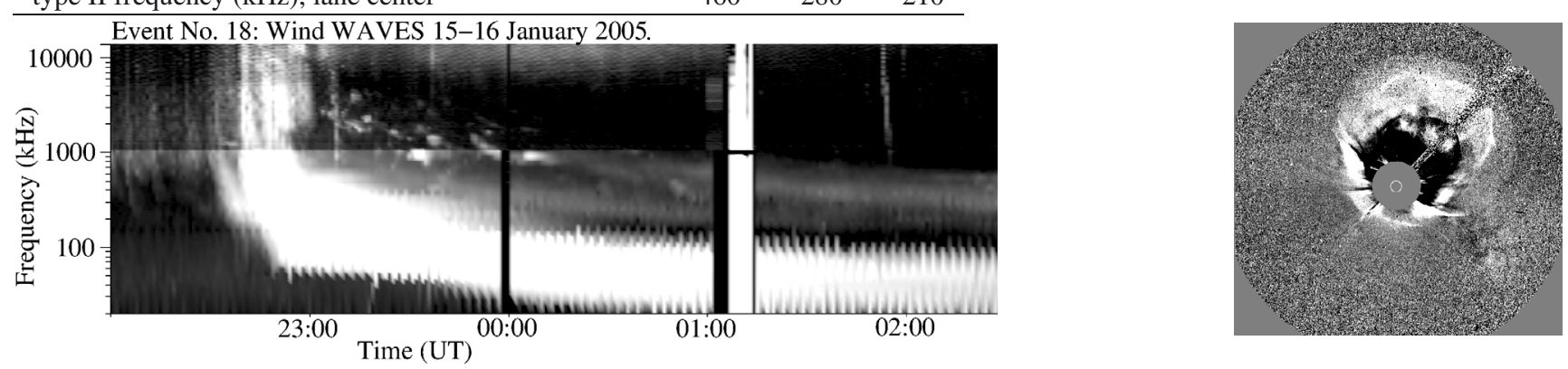

Fig. 19. Estimated CME and type II burst heights (lower emission lane, the two separate lanes overlap after 01:00 UT) for the 15-16 January 2005 event (Event No. 18). The the lanes overlap after 01:00 UT. The Wind WAVES frequency range is as in Fig. 2. The LASCO C3 image difference image shows the CME at 00:18 UT, at height $24.9 R_{\odot}$, at central position angle of $325^{\circ}$ (counterclockwise from the top). This was a full halo CME. Estimated first SEP injection time was at 22:52 \pm 5 UT. 
A\&A 558, A7 (2013)

\begin{tabular}{|c|c|c|c|c|c|}
\hline ent No. 19 & & & & & \\
\hline Time (UT) & $12: 32$ & $12: 38$ & 13:08 & $13: 38$ & $14: 38$ \\
\hline CME height $\left(R_{\odot}\right)$ & 5.2 & 6.4 & & 15.3 & 23.6 \\
\hline type II height $\left(R_{\odot}\right)$, hybrid model & & & 12.6 & 16.5 & 24.0 \\
\hline type II frequency & & & 700 & 460 & 270 \\
\hline
\end{tabular}
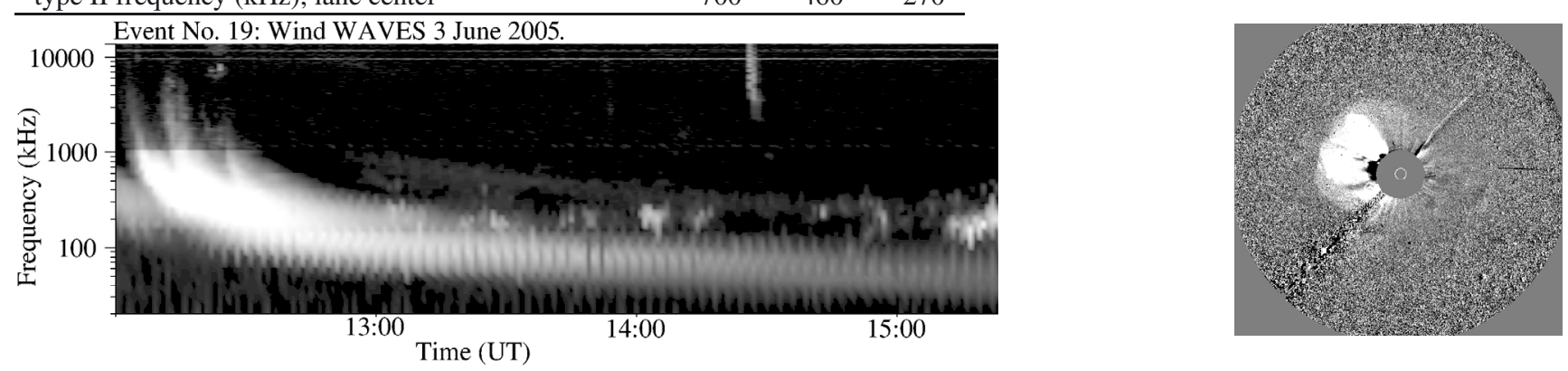

Fig. 20. Estimated CME and type II burst heights for the 3 June 2005 event (Event No. 19). The Wind WAVES frequency range is as in Fig. 2. The LASCO C3 image difference image shows the CME at 13:38 UT, at height $15.3 R_{\odot}$, at central position angle of $71^{\circ}$ (counterclockwise from the top). This was a full halo CME. SEP injection time analysis was not possible.
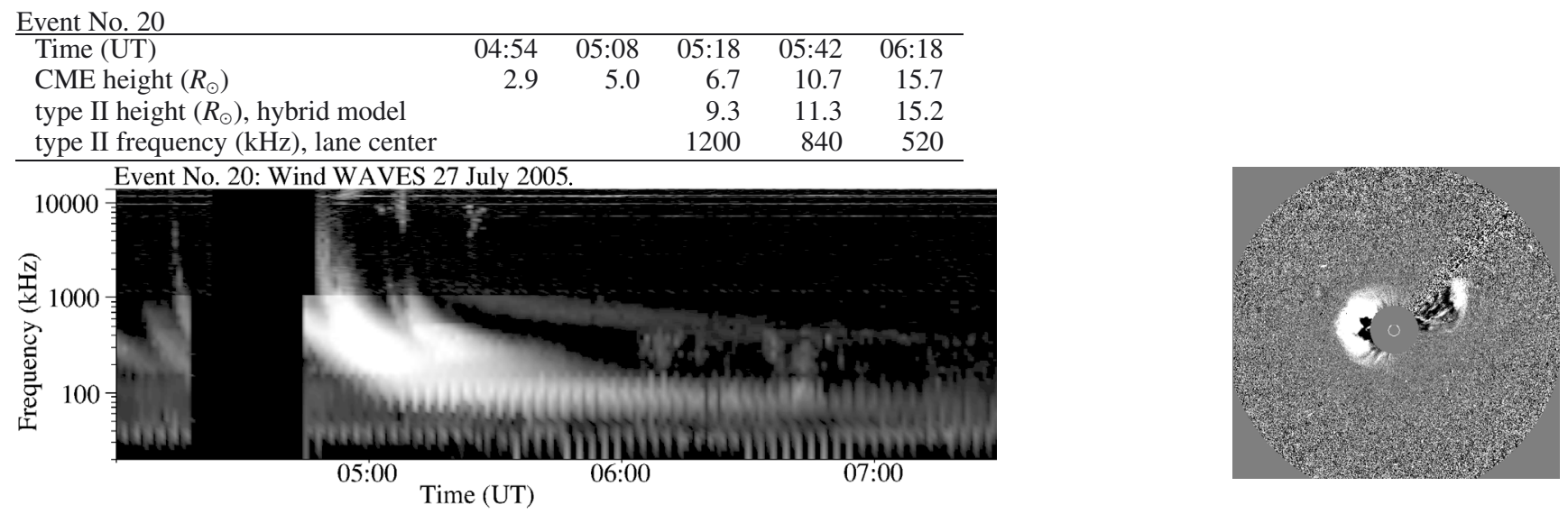

Fig. 21. Estimated CME and type II burst heights for the 27 July 2005 event (Event No. 20). The Wind WAVES frequency range is as in Fig. 2. The LASCO C3 image difference image shows the CME at 05:42 UT, at height $10.7 R_{\odot}$, at central position angle of $86^{\circ}$ (counterclockwise from the top). This was a full halo CME. SEP injection time analysis was not possible.

\begin{tabular}{lrrrrr} 
Event No. 21 & & & & & \\
\hline Time (UT) & $06: 50$ & $07: 00$ & $07: 33$ & $07: 53$ & $08: 52$ \\
CME height $\left(R_{\odot}\right)$ & 5.6 & 7.4 & 13.8 & 17.1 & 26.2 \\
type II height $\left(R_{\odot}\right)$, hybrid model & 8.2 & 10.4 & 13.3 & & \\
type II frequency $(\mathrm{kHz})$, lane center & 1500 & 1000 & 650 & & \\
\hline
\end{tabular}
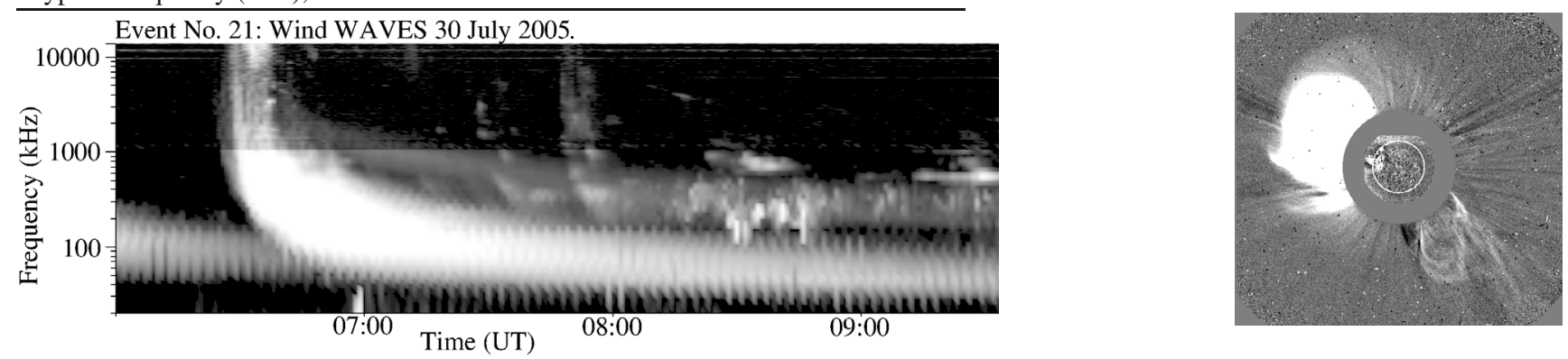

Fig. 22. Estimated CME and type II burst heights for the 30 July 2005 event (Event No. 21). The Wind WAVES frequency range is as in Fig. 2. The LASCO C2 image difference image shows the CME at 06:50 UT, at height 5.6 $R_{\odot}$, at central position angle of $48^{\circ}$ (counterclockwise from the top). This was a full halo CME. SEP injection time analysis was not possible. 
S. Pohjolainen et al.: Origin of wide-band IP type II bursts

\begin{tabular}{|c|c|c|c|c|c|c|}
\hline & & & & & & \\
\hline Time (UT) & $19: 48$ & $20: 00$ & $20: 18$ & $20: 42$ & $21: 18$ & $21: 42$ \\
\hline CME height $\left(R_{\odot}\right)$ & 3.1 & 5.6 & 9.6 & 14.9 & 20.9 & 25.3 \\
\hline type II height $\left(R_{\odot}\right)$, hybrid model & & 5.9 & 8.4 & 13.6 & & 25.0 \\
\hline type II frequency $(\mathrm{kHz})$, lane center & & 2800 & 1300 & 620 & & 260 \\
\hline
\end{tabular}

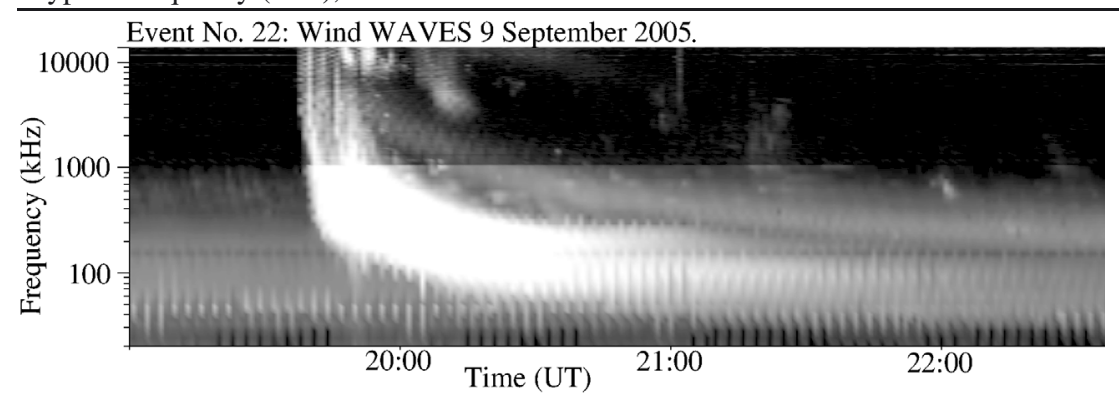

Fig. 23. Estimated CME and type II burst heights for the 9 September 2005 event (Event No. 22). The Wind WAVES frequency range is as in Fig. 2. The LASCO C2 image difference image shows the CME at 20:00 UT, at height 5.6 $R_{\odot}$, at central position angle of $112^{\circ}$ (counterclockwise from the top). This was a full halo CME. SEP injection time analysis was not possible.

\begin{tabular}{|c|c|c|c|c|c|c|c|}
\hline No. 23 & & & & & & & \\
\hline Time (UT) & $21: 52$ & $22: 00$ & $22: 07$ & $22: 15$ & $22: 37$ & $23: 41$ & $00: 00$ \\
\hline CME height $\left(R_{\odot}\right)$ & 4.0 & 5.4 & 7.0 & 8.6 & 12.8 & 21.8 & \\
\hline type II height $\left(R_{\odot}\right)$, hybrid model & 4.9 & 6.3 & 7.3 & 8.3 & 12.1 & 20.0 & 21.5 \\
\hline type II frequen & 4200 & 2500 & 1900 & 1400 & 750 & 340 & 310 \\
\hline
\end{tabular}

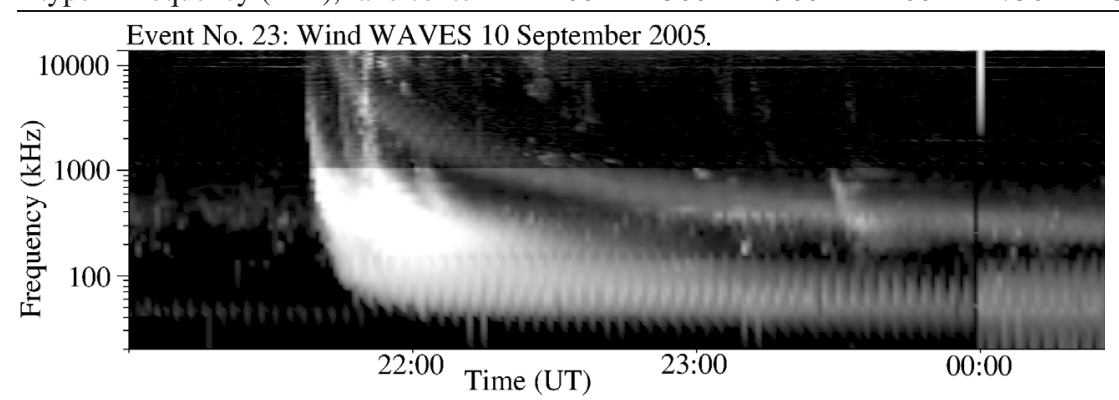

Fig. 24. Estimated CME and type II burst heights for the 10 September 2005 event (Event No. 23). The Wind WAVES frequency range is as in Fig. 2. The LASCO C2 image difference image shows the CME at 22:00 UT, at height 5.4 $R_{\odot}$, at central position angle of $120^{\circ}$ (counterclockwise from the top). This was a full halo CME. SEP injection time analysis was not possible.

\begin{tabular}{|c|c|c|c|c|c|c|c|c|}
\hline & & & & & & & & \\
\hline Time ( & $20: 00$ & $20: 18$ & $20: 30$ & $21: 18$ & $21: 42$ & $22: 06$ & $22: 18$ & $02: 30$ \\
\hline CME height $\left(R_{\odot}\right)$ & 2.4 & 5.7 & 8.4 & 17.3 & 21.3 & 25.4 & 27.6 & \\
\hline type II height $\left(R_{\odot}\right)$, hybric & & & & & 11.1 & 13.1 & 14.0 & 22.5 \\
\hline type II frequency $(\mathrm{kHz})$, lane center & & & & & 880 & 660 & 600 & 280 \\
\hline
\end{tabular}

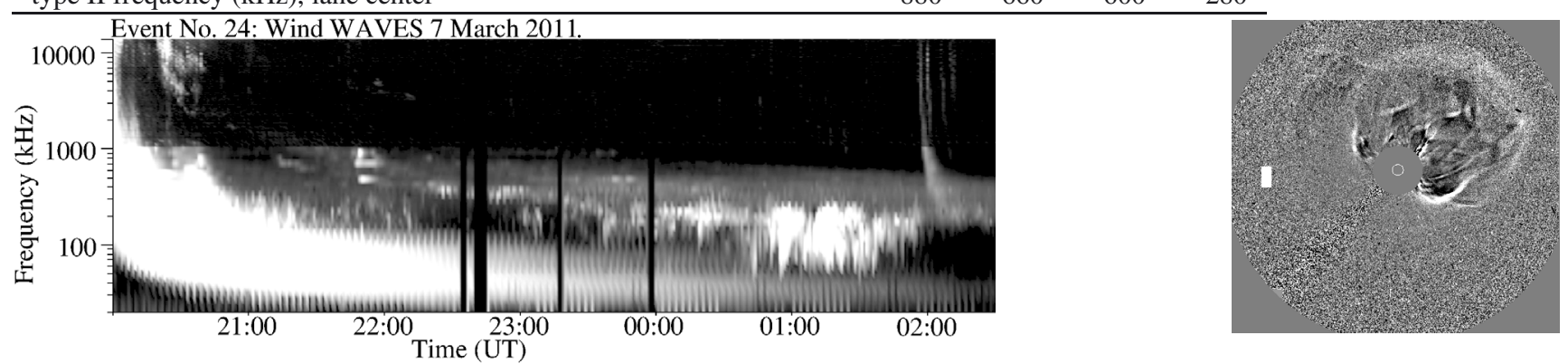

Fig. 25. Estimated CME and type II burst heights for the 7 March 2011 event (Event No. 24). The Wind WAVES frequency range is as in Fig. 2. The LASCO C3 image difference image shows the CME at 22:06 UT, at height $25.4 R_{\odot}$, at central position angle of $314^{\circ}$ (counterclockwise from the top). This was a full halo CME. Estimated first SEP injection time was at 20:27 \pm 6 UT. 
A\&A 558, A7 (2013)

\begin{tabular}{|c|c|c|c|c|c|c|c|c|}
\hline Event No. 25 & & & & & & & & \\
\hline Time (UT) & $10: 48$ & $11: 06$ & $11: 30$ & $12: 06$ & $12: 30$ & $13: 30$ & $14: 00$ & $15: 30$ \\
\hline CME height $\left(R_{\odot}\right)$ & 3.0 & 6.2 & 10.4 & 16.5 & 20.4 & 28.7 & & \\
\hline type II height $\left(R_{\odot}\right)$, hybrid model & & & & & 12.6 & 15.6 & 16.7 & 22.0 \\
\hline type II frequency $(\mathrm{kHz})$, lane center & & & & & 700 & 500 & 450 & 300 \\
\hline
\end{tabular}

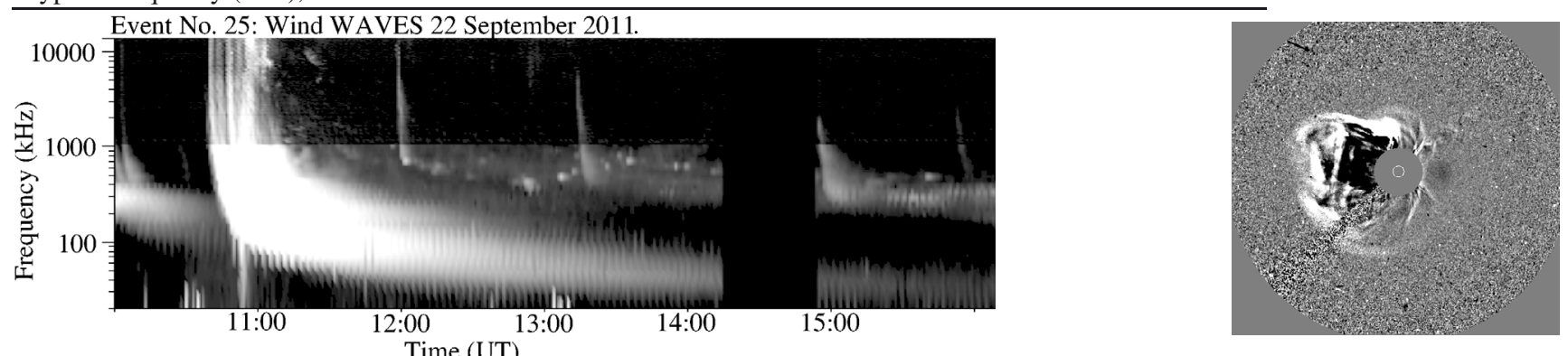

Fig. 26. Estimated CME and type II burst heights for the 22 September 2011 event (Event No. 25). The Wind WAVES frequency range is as in Fig. 2. The LASCO C3 image difference image shows the CME at 12:30 UT, at height $20.4 R_{\odot}$, at central position angle of $71^{\circ}$ (counterclockwise from the top). This was a full halo CME. SEP injection time analysis was not possible. 
Table 4. Estimated SEP injection times for the analyzed events Nos. 1-25.

\begin{tabular}{|c|c|c|c|c|}
\hline No. & $\begin{array}{l}\text { Source } \\
\text { pos. }\end{array}$ & $\begin{array}{c}\text { Injection } \\
\text { time (UT) } \\
\text { VDA } \\
\text { method }\end{array}$ & $\begin{array}{c}\text { Injection } \\
\text { time (UT) } \\
\text { fixed path } \\
1.2 \mathrm{AU}\end{array}$ & Description \\
\hline 1 & E23 & $\mathrm{U} 10: 31 \pm 8$ & $10: 59$ & $\begin{array}{l}\text { At high energies the main event rose directly from the low background, but a precursor } \\
\text { event was visible in the mid-range of energies causing possible systematic errors in the } \\
\text { VDA results. Apparent path length with VDA } 2.20 \pm 0.08 \text { AU. }\end{array}$ \\
\hline 2 & W-limb & $\mathrm{U} 07: 56 \pm 48$ & 07:35 & $\begin{array}{l}\text { On the tail of an earlier event and onset clearly seen only at high energies }(>30 \mathrm{MeV}) \text {. } \\
\text { VDA analysis was done with only } 5 \text { out of } 20 \text { channels, giving apparent path length } \\
2.1 \pm 0.34 \text { AU. }\end{array}$ \\
\hline 3 & E20 & $\mathrm{U} 14: 20 \pm 11$ & $15: 23$ & $\begin{array}{l}\text { On the tail of a series of events, apparent path length } 3.24 \pm 0.12 \mathrm{AU} \text {. There are great } \\
\text { uncertainties in the onset times due to the slow rise. }\end{array}$ \\
\hline 4 & W29 & $13: 05 \pm 9$ & $14: 12$ & $\begin{array}{l}\text { Clear event with not much background, lowest energy channels not usable ( } 14 \text { channels } \\
\text { used for VDA). Apparent path length } 2.86 \pm 0.10 \mathrm{AU} \text {. }\end{array}$ \\
\hline 5 & W37 & & 06:09 & $\begin{array}{l}\text { There may be a rise at the time of maximum of an earlier event, but VDA was not } \\
\text { possible. }\end{array}$ \\
\hline 6 & W22 & $\mathrm{U} 00: 15 \pm 11$ & 00:19 & $\begin{array}{l}\text { Clear event on low background, but intermittency of the data and some precursors at the } \\
\text { beginning make VDA uncertain ( } 8 \text { channels used). Path length } 4.82 \pm 0.09 \mathrm{AU} \text {. }\end{array}$ \\
\hline 7 & W37 & & & Nothing new is seen at the time of the flare, on top of an earlier event. \\
\hline 8 & W65 & $\mathrm{U} 02: 30 \pm 4$ & $02: 27$ & $\begin{array}{l}\text { On the tail of earlier events and a precursor at }>12 \mathrm{MeV} \text {. Using all } 20 \text { energy chan- } \\
\text { nels gives injection time } 01: 54 \pm 11 \mathrm{~min} \text {, path length } 2.82 \pm 0.16 \mathrm{AU} \text {. Using only the } \\
8 \text { highest energy channels gives } 02: 30 \pm 4 \text { min, path length } 1.36 \pm 0.03 \mathrm{AU} \text {. }\end{array}$ \\
\hline 9 & E55 & & $08: 21$ & $\begin{array}{l}\text { Event occurred on an elevated, fluctuating background, and onset was closely simulta- } \\
\text { neous at all energy channels }<55 \mathrm{MeV} \text {. This may indicate that the shock driven by the } \\
\text { CME did not reach magnetic connection to SOHO until close to the spacecraft. }\end{array}$ \\
\hline 10 & E15 & & & $\begin{array}{l}\text { Very small enhancement was observed over the quiet-time background up to } \sim 15 \mathrm{MeV} \text {, } \\
\text { but the event was so small that an integration time of } 10 \text { min was required to observe it } \\
\text { (statistics at shorter integration times were too low to see anything significant). }\end{array}$ \\
\hline 11 & W47 & $04: 06 \pm 6$ & $04: 40$ & $\begin{array}{l}\text { Clear SEP event observed at all energies }>1.5 \mathrm{MeV} \text {, high background at low energies } \\
(<10 \mathrm{MeV}) \text {. Apparent path length } 2.19 \pm 0.08 \mathrm{AU} \text {. }\end{array}$ \\
\hline 12 & W33 & $15: 04 \pm 6$ & $15: 33$ & $\begin{array}{l}\text { On the tail of an earlier event, strongly fluctuating rise at energies }<10 \mathrm{MeV} \text {. Apparent } \\
\text { path length } 2.00 \pm 0.06 \mathrm{AU} \text {. }\end{array}$ \\
\hline 13 & W-limb & & & $\begin{array}{l}\text { On the tail of an earlier event. At energies }<10 \mathrm{MeV} \text { the intensities were steadily falling, } \\
\text { but at higher energies a small slow rise was observed. VDA was not possible because } \\
\text { definite SEP onset times could not be determined. }\end{array}$ \\
\hline 14 & W-limb & & & $\begin{array}{l}\text { On the tail of earlier events. In the energy range of }<6 \mathrm{MeV} \text { an enhancement was ob- } \\
\text { served. Probably due to the high background, the } \mathrm{SEP} \text { event was seen to start at succes- } \\
\text { sively later times with increasing energy. }\end{array}$ \\
\hline 15 & E42 & & & $\begin{array}{l}\text { Very small increases of intensities above a high background, VDA did not allow the } \\
\text { injection time to be determined reliably. }\end{array}$ \\
\hline 16 & E03 & & & $\begin{array}{l}\text { Precursor at low energies, above a low background. Because of the precursor, the in- } \\
\text { tensities at the highest energies seemed to rise above the background later than at low } \\
\text { energies and the VDA did not give reasonable injection time results. }\end{array}$ \\
\hline 17 & E04 & $06: 20 \pm 4$ & $06: 44$ & $\begin{array}{l}\text { Clear SEP event on a low background, reaching energies of } \sim 100 \mathrm{MeV} \text {. Apparent path } \\
\text { length } 1.48 \pm 0.05 \mathrm{AU} \text {. }\end{array}$ \\
\hline 18 & W05 & $22: 52 \pm 5$ & $22: 11$ & $\begin{array}{l}\text { Completely masked at lowest energies }(<3 \mathrm{MeV}) \text { by an earlier event, but at higher } \\
\text { energies visible up to } \sim 100 \mathrm{MeV} \text {. Apparent path length } 2.32 \pm 0.06 \mathrm{AU} \text {. }\end{array}$ \\
\hline 19 & E-limb & & & $\begin{array}{l}\text { Slightly elevated falling background from an earlier event, modest slow rise was visible } \\
\text { up to } \sim 60 \mathrm{MeV} \text {. Statistics were so low that VDA was not possible. }\end{array}$ \\
\hline 20 & E-limb & & & $\begin{array}{l}\text { SEP intensities had been slowly rising for two days and a small additional jump in } \\
\text { intensities was observed. The event seemed to have only weak velocity dispersion and } \\
\text { a data gap made detailed analysis very uncertain. }\end{array}$ \\
\hline 21 & E60 & & & $\begin{array}{l}\text { No association to any new SEP event. Occurred during the falling phase of an earlier } \\
\text { event. A long gap in the ERNE data prevents identification of any possible later en- } \\
\text { hancements. }\end{array}$ \\
\hline 22 & E67 & & & $\begin{array}{l}\text { Intensities at low energies had been slowly rising for two days. No additional enhance- } \\
\text { ment at any energy was observed which could be associated with the flare and CME. }\end{array}$ \\
\hline 23 & E47 & & & $\begin{array}{l}\text { Slowly rising background, same as in event } 22 \text {. At high energies a new slow small rise } \\
\text { was observed but it is not clear at all that this was related to the flare/CME. }\end{array}$ \\
\hline 24 & W-limb & $20: 27 \pm 6$ & $20: 38$ & $\begin{array}{l}\text { Rapid and clear rise observed up to } \sim 100 \mathrm{MeV} \text {, with a well-separated precursor at } \\
>10 \mathrm{MeV} \text {. Apparent path length of } 1.85 \pm 0.08 \mathrm{AU} \text {. }\end{array}$ \\
\hline 25 & E-limb & & & $\begin{array}{l}\text { Several ERNE data gaps, no SEP event could be identified associated with the } \\
\text { flare/CME event. }\end{array}$ \\
\hline
\end{tabular}

